\title{
A transformation rule for the index of commuting operators
}

\author{
Jens Kaad*and Ryszard Nest**
}

\begin{abstract}
In the setting of several commuting operators on a Hilbert space one defines the notions of invertibility and Fredholmness in terms of the associated Koszul complex. The index problem then consists of computing the Euler characteristic of such a special type of Fredholm complex.

In this paper we investigate transformation rules for the index under the holomorphic functional calculus. We distinguish between two different types of index results:

1) A global index theorem which expresses the index in terms of the degree function of the "symbol" and the locally constant index function of the "variables".

2) A local index theorem which computes the Euler characteristic of a localized Koszul complex near a common zero of the "symbol".

Our results apply to the example of Toeplitz operators acting on both Bergman spaces over pseudoconvex domains and the Hardy space over the polydisc.

The local index theorem is fundamental for future investigations of determinants and torsion of Koszul complexes.
\end{abstract}

Mathematics Subject Classification (2010). 47A13, 47A53; 46A60, 32A10, 13D07, 14C17.

Keywords. Index theory, Koszul complex, commuting operators, holomorphic functional calculus, localization, multiplicity.

\section{Introduction}

The purpose of this paper is to prepare the stage for the study of determinants and torsion of complexes associated to $n$-tuples $A=\left(A_{1}, \ldots, A_{n}\right)$ of commuting operators on a Hilbert space $\mathscr{H}$ (cf. [8]). Let us recall the case of two commuting Toeplitz operators $\left(T_{f}, T_{g}\right)$, as studied by Carey and Pincus ([1]). The torsion $\operatorname{Tor}(f, g)$ of such a pair of operators can be identified as an invariant of the class of the Loday product $f \times g$ in the group $K_{2}^{a l g}\left(\mathcal{O}_{\mathbb{D}}\right)$, the algebraic K-theory of the functions holomorphic in the unit disc. It can be identified with what is known as the

\footnotetext{
* The first author is supported by the Danish Carlsberg Foundation.

** The second author is partially supported by the Danish National Research Foundation (DNRF) through the Centre for Symmetry and Deformation.
} 
Tate tame symbol of the pair $(f, g)$ and it's value is given by the residue formula :

$$
\operatorname{Tor}(f, g)=\exp \left(\frac{1}{2 \pi i} \int_{\partial \mathbb{D}} \log f d(\log g)\right)= \pm \frac{\prod_{g(\lambda)=0,|\lambda|<1} f(\lambda)^{\operatorname{deg}_{\lambda} g}}{\prod_{f(\mu)=0,|\mu|<1} g(\mu)^{\operatorname{deg}_{\mu} f}} .
$$

The exponents in the expression on the right hand side stand for the multiplicities of zeroes of the functions $f$ and $g$ in the interior of the unit disc. These local multiplicities are given by local winding numbers of $f$ and $g$ and can be interpreted as a localized version of the indices of the Toeplitz operators $T_{f}$ and $T_{g}$, while the overall sign is given by the parity of the product of the standard (Fredholm) indices of the operators $T_{f}$ and $T_{g}$.

For an arbitrary n-tuple $A=\left(A_{1}, \ldots, A_{n}\right)$ the associated torsion is expected to involve, in analogy to the above case, a product of exponentials with the exponents given by local indices of the $n$-tuple as defined below and whose computation is the main subject of this paper.

The type of index theorems in our setting can be thought of as a transformation rule for the index under Taylor's holomorphic functional calculus. Loosely speaking we start out with a commuting tuple of "variables" $A$ together with a holomorphic "symbol" $g: \operatorname{Sp}(A) \rightarrow \mathbb{C}^{m}$ on the Taylor spectrum of $A$ (cf. [20]). Under the condition that the commuting tuple $g(A)$ is Fredholm we distinguish between two different index results:

(1) A global index theorem which expresses the Fredholm index of $g(A)$ in terms of the locally constant index function $\lambda \mapsto \operatorname{Ind}(A-\lambda)$ associated with the coordinates $A$ and local degrees of the symbol $g$ near the set of zeroes.

(2) A local index theorem which expresses the Euler characteristic of a localized Koszul complex at a common zero $\lambda$ for the symbol $g$ in terms of the local degree (or intersection multiplicity) $\operatorname{deg}_{\lambda}(g)$ and the index $\operatorname{Ind}(A-\lambda)$.

Our basic tool is the study of the localizations of $\mathscr{H}$ as a module over the ring $\mathcal{O}(\mathrm{Sp}(A))$ of germs of holomorphic functions on the Taylor spectrum of $A$.

Before we provide more details on the above results, let us recall some information on the multivariable holomorphic calculus.

Naturally associated to a commuting $n$-tuple $A=\left(A_{1}, \ldots, A_{n}\right)$ is a Koszul complex (see Definition 2.1):

$$
K_{*}(A, \mathscr{H})=\left(\mathscr{H} \otimes \Lambda_{*} \mathbb{C}^{n}, d_{A}\right)
$$

where $\Lambda_{*} V$ stands for the exterior algebra of $V$ and the boundary operator is of the form $d_{A}=\sum_{i} A_{i} \otimes \epsilon_{i}{ }^{*}$ and the $\epsilon_{i}^{*}$ 's are interior multiplication operators with the standard basis vectors in $\mathbb{C}^{n} . A$ is called invertible if $K_{*}(A, \mathscr{H})$ is contractible and it is called Fredholm if the homology of $K_{*}(A, \mathscr{H})$ is finite dimensional. When $A$ 
is Fredholm, the index is minus the Euler characteristic of the Koszul complex,

$$
\operatorname{Ind}(A):=-\sum_{i}(-1)^{i} \operatorname{Dim}_{\mathbb{C}}\left(H_{i}(A, \mathscr{H})\right) .
$$

In the case where $n=1$, these definitions coincide with the usual terminology.

An analogue of the spectrum of a single operator was introduced by Taylor (see [20]). The Taylor spectrum $\operatorname{Sp}(A)$ of $A$ is defined as the set of $\lambda \in \mathbb{C}^{n}$ such that $A-\lambda$ is not invertible. The Taylor spectrum is a compact and non-empty set. The main property of this spectrum is the fact that it supports the multivariable holomorphic functional calculus, i. e. a continuous homomorphism

$$
\mathcal{O}(\operatorname{Sp}(A)) \rightarrow \mathscr{L}(\mathscr{H})
$$

from the germs of holomorphic functions on $\operatorname{Sp}(A)$ to bounded operators on $\mathscr{H}$ such that the coordinate functions $z_{i}$ are mapped to the operators $A_{i}$. In particular, given a holomorphic map $g=\left(g_{1}, \ldots, g_{m}\right): \mathrm{Sp}(A) \rightarrow \mathbb{C}^{m}$ one obtains a new commuting tuple $g(A)=\left(g_{1}(A), \ldots g_{m}(A)\right)$.

Under the condition that $g(A)$ is Fredholm there is thus an associated index $\operatorname{Ind}(g(A))$. The intuition which lies behind our index theorems is that this quantity should be expressible in terms of the local behaviour of the symbol $g: \operatorname{Sp}(A) \rightarrow \mathbb{C}^{m}$ near its singularities. Since $g$ is holomorphic, the singularities constitute the set of common zeroes

$$
Z(g)=\left\{\lambda \in \operatorname{Sp}(A) \mid g_{1}(\lambda)=\cdots=g_{m}(\lambda)=0\right\} .
$$

It turns out that the Fredholm assumption implies that this set is finite (Theorem 3.3).

In order to explain the local quantity of our index theorems further, assume that $n=m$, thus that the number of variables in $A=\left(A_{1}, \ldots, A_{n}\right)$ is the same as the number of functions in $g=\left(g_{1}, \ldots, g_{m}\right)$. Let us also fix an element $\lambda \in \operatorname{Sp}(A)^{\circ}$ in the interior of the spectrum. The notation $\mathcal{O}_{\lambda}$ will refer to the unital algebra over $\mathbb{C}$ of power series convergent near $\lambda$. It follows from the finiteness of the set of common zeroes $Z(g)$ that the quotient vector space $\mathcal{O}_{\lambda} / g \mathcal{O}_{\lambda}$ has finite dimension, where $g \mathcal{O}_{\lambda}$ denotes the ideal generated by the functions $g_{1}, \ldots, g_{n}$ in $\mathcal{O}_{\lambda}$. We may thus define the local degree (or intersection multiplicity) of the symbol $g$ near $\lambda$ as the non-negative integer

$$
\operatorname{deg}_{\lambda}(g):=\operatorname{Dim}_{\mathbb{C}}\left(\mathcal{O}_{\lambda} / g \mathcal{O}_{\lambda}\right) .
$$

Remark that $\operatorname{deg}_{\lambda}(g)$ is non-trivial if and only if $\lambda \in Z(g) \cap \operatorname{Sp}(A)^{\circ}$.

The global index theorem can now be stated as follows.

Theorem 1.1. Suppose that $g(A)$ is Fredholm. In this case the set $Z(g)=$ $g^{-1}(\{0\}) \cap \operatorname{Sp}(A)$ is finite and

$$
\operatorname{Ind}(g(A))= \begin{cases}\sum_{\lambda \in Z(g)} \operatorname{deg}_{\lambda}(g) \operatorname{Ind}(A-\lambda) & \text { when } n=m \\ 0 & \text { when } n<m\end{cases}
$$


We remark that the global index theorem was proved by Eschmeier and Putinar in [4, Theorem 10.3.13]. Their techniques are however different from ours as they rely on the acyclicity properties of Čech complexes associated with Stein open coverings of the spectrum. See also [13] and [11]. The type of localization which we apply is of a more algebraic nature.

In order to state our local index theorem, we will now introduce the local indices using the localization procedure alluded to above. From now on we will suppose that $g(A)$ is Fredholm.

Let $\mathcal{O}(\operatorname{Sp}(A))$ denote the ring of germs of holomorphic functions on $\operatorname{Sp}(A)$. The holomorphic functional calculus gives $\mathscr{H}$ the structure of a $\mathcal{O}(\mathrm{Sp}(A))$-module. For each $\lambda \in \operatorname{Sp}(A)$ we consider the localized module $\mathscr{C}_{\lambda}$ at the prime ideal $\mathfrak{p}_{\lambda}=\{f \in$ $\mathcal{O}(\operatorname{Sp}(A)) \mid f(\lambda)=0\}$. The local index at $\lambda$ is then defined as minus the Euler characteristic of the localized Koszul complex $K_{*}\left(g, \mathscr{H}_{\lambda}\right)$,

$$
\operatorname{Ind}_{\lambda}(g(A)):=-\sum_{i}(-1)^{i} \operatorname{Dim}_{\mathbb{C}}\left(H_{i}\left(g, \mathscr{H}_{\lambda}\right)\right) .
$$

Note that the homology groups are finite dimensional as a consequence of the Fredholmness of $g(A)$.

Another way of constructing the localized modules $H_{*}\left(g, \mathscr{H}_{\lambda}\right)$ consists of decomposing the finite dimensional homology groups $H_{*}(g(A), \mathscr{H})$ into the generalized eigenspaces of the commuting tuple $\left(H_{*}\left(A_{1}\right), \ldots, H_{*}\left(A_{n}\right)\right)$ induced by the action of the commuting tuple $A$. See Proposition 4.8.

An important feature of the homology groups $H_{*}\left(g, \mathscr{H}_{\lambda}\right)$ is that they can be turned into modules over the ring $\mathcal{O}_{\lambda}$ of power series convergent near $\lambda \in \operatorname{Sp}(A)$. More precisely, there is a homomorphism $\mathcal{O}_{\lambda} \rightarrow \mathscr{L}\left(H_{i}\left(g, \mathscr{H}_{\lambda}\right)\right)$, for each $i \in\{0, \ldots, m\}$, which makes the diagram

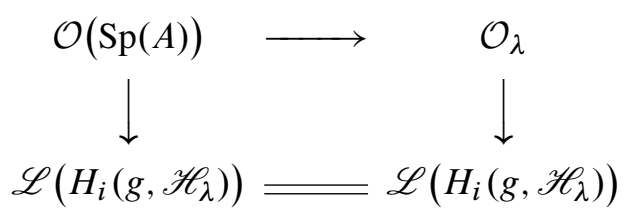

commute. Here the upper horizontal map is the restriction homomorphism and the left vertical map is the homomorphism associated with the natural action of $\mathcal{O}(\operatorname{Sp}(A))$ on $H_{i}\left(g, \mathscr{H}_{\lambda}\right)$.

The relation between the local indices and the global index can then be described by the summation formula:

$$
\operatorname{Ind}(g(A))=\sum_{\lambda \in Z(g)} \operatorname{Ind}_{\lambda}(g(A)) .
$$

Our local index theorem can now be stated as follows. 
Theorem 1.2. Suppose that $g(A)$ is Fredholm and that $g(\lambda)=0$. Then the homology groups $H_{*}\left(g, \mathscr{H}_{\lambda}\right)$ are finite dimensional and

$$
\operatorname{Ind}_{\lambda}(g(A))=\operatorname{deg}_{\lambda}(g) \operatorname{Ind}(A-\lambda)
$$

The main step in the proof of the local index theorem is an analytic analogue of the algebraic localization procedure described above. Passing to the analytic localization at a point $\lambda \in \mathbb{C}^{n}$ amounts to taking the tensor product with a Hilbert space which comes equipped with a commuting tuple $B$ with spectrum a small closed ball with center $\lambda, \operatorname{Sp}(B)=\operatorname{cl}\left(\mathbb{B}_{\varepsilon}(\lambda)\right)$. Furthermore, the commuting tuple $\lambda-B$ is required to be Fredholm with $\operatorname{Ind}(\lambda-B) \neq 0$. When computing indices it is then necessary to "restrict to the diagonal" by taking the union with the commuting tuple $A \otimes 1-1 \otimes B$. The reason for applying this procedure is that the local index is not a homotopy invariant.

One consequence of the results needed for studying the above analytic localization is the following reciprocity result.

Theorem 1.3. Suppose that $A$ and $B$ are two commuting tuples of the same length. Let $g: \operatorname{Sp}(A) \cup \operatorname{Sp}(B) \rightarrow \mathbb{C}^{m}$ be holomorphic. Suppose that $g(A)$ and $g(B)$ are Fredholm and that the sets $Z(g) \cap \mathrm{Sp}(A) \cap \mathrm{Sp}_{\mathrm{ess}}(B)$ and $Z(g) \cap \mathrm{Sp}(B) \cap \mathrm{Sp}_{\mathrm{ess}}(A)$ are empty. Then

$$
\sum_{\mu \in Z(g) \cap \mathrm{Sp}(B)} \operatorname{Ind}(\mu-A) \cdot \operatorname{Ind}_{\mu}(g(B))=\sum_{\lambda \in Z(g) \cap \mathrm{Sp}(A)} \operatorname{Ind}_{\lambda}(g(A)) \cdot \operatorname{Ind}(\lambda-B) .
$$

The structure of this paper is as follows.

In the next section we collected the general definitions and results connected with the multivariable holomorphic functional calculus and some results from homological algebra related to Koszul complexes (see subsection 2.4) which are useful later on.

In Section 3 we collected some basic results on the behaviour of Fredholm spectra under holomorphic maps. The algebraic localization results are proved in Section 4. The local index theorem for regular zeroes of $g$ is proved in Section 5. A simple proof of the global index theorem is given in Section 6 (see Theorem 6.6). The analytic localization is the subject of Section 7. The local index theorem is proved in Section 8. We end the paper by illustrating the applicability of our results by computing indices for Toeplitz operators acting on Hardy spaces and Bergman spaces over higher dimensional domains.

Remark 1.4. Let us note that our "local index theorem" is of a somewhat different nature than the standard index theorems. The index theorems of Atiyah-Singer type represent the index of a, say, elliptic pseudodifferential operator $D$ in terms of an integral of a "local expression", to wit a differential form representing a characteristic class associated with the symbol of $D$. In our case the localization happens directly 
at the level of the tuple $A$ (or more precisely its Koszul complex). So, for a Toeplitz operator $T_{f}$ with a holomorphic symbol $f$ invertible on the boundary of the unit disc, one approach gives the integral of the characteristic class $\frac{1}{2 \pi i} f^{-1} d f$ over the boundary of the unit disc while the second gives the sum of orders of poles of $f^{-1}$ in the interior of $\mathbb{D}$.

\section{Preliminaries}

Let $A=\left(A_{1}, \ldots, A_{n}\right)$ be a commuting tuple of linear operators on a vector space $V$ over the complex numbers $\mathbb{C}$.

2.1. Koszul homology. Let $\Lambda\left(\mathbb{C}^{n}\right)=\oplus_{k=1}^{n} \Lambda_{k}\left(\mathbb{C}^{n}\right)$ where, as a vector space, $\Lambda_{k}\left(\mathbb{C}^{n}\right)$ coincides with the usual exterior power $\Lambda^{k}\left(\mathbb{C}^{n}\right)$. For a subset $I \subseteq$ $\{1, \ldots, n\}$ we use the notation $e_{I}:=e_{i_{1}} \wedge \ldots \wedge e_{i_{k}} \in \Lambda_{k}\left(\mathbb{C}^{n}\right), i_{1}<\ldots<i_{k}$. We will give $\Lambda\left(\mathbb{C}^{n}\right)$ the structure of a Hilbert space in which the basis $\left\{e_{I}\right\}_{I \subseteq\{1, \ldots, n\}}$ is orthonormal. For each $i \in\{1, \ldots, n\}$ we set

$$
\varepsilon_{i}: \Lambda\left(\mathbb{C}^{n}\right) \rightarrow \Lambda\left(\mathbb{C}^{n}\right) ; e_{I} \mapsto e_{i} \wedge e_{I}
$$

The adjoint of this exterior multiplication operator will be denoted by $\varepsilon_{i}^{*}: \Lambda\left(\mathbb{C}^{n}\right) \rightarrow$ $\Lambda\left(\mathbb{C}^{n}\right)$.

Definition 2.1. The Koszul complex $K_{*}(A, V)$ of $A$ on $V$ is the chain complex

$$
\left(V \otimes \Lambda_{*}\left(\mathbb{C}^{n}\right), d_{A}\right),
$$

where $d_{A}:=\sum_{i=1}^{n} A_{i} \otimes \varepsilon_{i}^{*}$. We will denote the homology groups of $K_{*}(A, V)$ by $H_{*}(A, V)$ and refer to these vector spaces as the Koszul homology groups of $A$. We will set $H(A, V):=\oplus_{k=0}^{n} H_{k}(A, V)$.

The tuple $A$ is said to be invertible if $H(A, V)=\{0\}$.

Remark 2.2. While $\Lambda\left(\mathbb{C}^{n}\right)$ coincides with the usual exterior algebra of $\mathbb{C}^{n}$, we use $\Lambda_{*}$ instead of the more usual $\Lambda^{*}$ to conform with the standard notation from homological algebra for homological complexes.

The letter " $\mathrm{K}$ " will, in a mathematical context, refer to the Koszul complex throughout this paper. Since we will not use K-theory in this paper, this should not cause any confusion.

The next definition is fundamental to the present text.

Definition 2.3. A commuting tuple $A$ is Fredholm when the Koszul homology groups $H_{*}(A, V)$ are finite dimensional. Given a Fredholm commuting tuple $A$, its Fredholm index is the integer

$$
\operatorname{Ind}(A):=\sum_{k=0}^{n}(-1)^{k+1} \operatorname{Dim}_{\mathbb{C}}\left(H_{k}(A, V)\right) .
$$


Let $X$ be a chain complex and recall that the mapping cone $C(\gamma, X)$ of a chain map $\gamma: X \rightarrow X$ is the chain complex

$X_{0} \stackrel{\left(\begin{array}{ll}d & \gamma\end{array}\right)}{\longleftarrow} X_{1} \oplus X_{0} \stackrel{\left(\begin{array}{cc}d & \gamma \\ 0 & -d\end{array}\right)}{\longleftarrow} \ldots \stackrel{\left(\begin{array}{cc}d & \gamma \\ 0 & -d\end{array}\right)}{\longleftarrow} X_{n} \oplus X_{n-1} \stackrel{\left(\begin{array}{c}\gamma \\ -d\end{array}\right)}{\longleftarrow} X_{n}$.

The homology groups of the mapping cone will be denoted below by $H_{*}(\gamma, X)$. These homology groups measure how far away $\gamma$ is from being a quasi-isomorphism. More precisely, there is a long exact sequence,

$\ldots \longleftarrow H_{k-1}(X) \longleftarrow H_{k}(\gamma, X) \longleftarrow H_{k}(X) \longleftarrow \gamma H_{k}(X) \longleftarrow \ldots$

Suppose that $B \in \operatorname{End}(V)$ commutes with $A_{1}, \ldots, A_{n}$ and let $A \oplus B$ denote the commuting $(n+1)$-tuple $\left(A_{1}, \ldots, A_{n}, B\right)$. Then $B$ defines a chain map $B: K_{*}(A, V) \rightarrow K_{*}(A, V)$. For future reference let us state the following observation.

Lemma 2.4. The Koszul complex $K_{*}(A \oplus B, V)$ is naturally isomorphic to the mapping cone $C\left(K_{*}(B), K_{*}(A, V)\right)$ of $K_{*}(B): K_{*}(A, V) \rightarrow K_{*}(A, V)$. In particular, if any of the $A_{i}$ 's is invertible, then $K_{*}(A, V)$ is contractible (i.e. its homology is zero).

Proof. For each $k \in\{0, \ldots, n+1\}$ we set

$$
\begin{aligned}
& \alpha_{k}: C_{k}\left(K_{*}(B), K_{*}(A, V)\right)=K_{k}(A, V) \oplus K_{k-1}(A, V) \rightarrow K_{k}(A \oplus B, V) \\
& \alpha_{k}:\left(\left(\xi \otimes e_{I}\right),\left(\eta \otimes e_{J}\right)\right) \mapsto \xi \otimes e_{I}+\eta \otimes e_{n+1} \wedge e_{J}
\end{aligned}
$$

It is not hard to verify that the collection $\left\{\alpha_{k}\right\}$ defines a chain isomorphism $\alpha: C\left(K_{*}(B), K_{*}(A, V)\right) \rightarrow K_{*}(A \oplus B, V)$.

The last statement of the lemma now follows from the long exact sequence in $(2.1)$

2.2. Commuting tuples on finite dimensional vector spaces. Let $A=\left(A_{1}, \ldots, A_{n}\right)$ be a commuting tuple of linear operators on a vector space $V$ of finite dimension over the complex numbers $\mathbb{C}$. The classical Lie theorem says that there exists a basis for $V$ in which all $A_{i}$ 's are simultaneously upper triangular. To be more precise, set, for each $\lambda \in \mathbb{C}^{n}$,

$$
V(\lambda):=\bigcup_{i_{1}, \ldots, i_{n} \in \mathbb{N}} \operatorname{Ker}\left(A_{1}-\lambda_{1}\right)^{i_{1}} \cap \ldots \cap \operatorname{Ker}\left(A_{n}-\lambda_{n}\right)^{i_{n}} \subseteq V
$$

and $\sigma(A)=\{\lambda \mid V(\lambda) \neq\{0\}\}$. The following holds. See for example [17, Chapter V, Theorem 5.1]. 
Theorem 2.5 (Lie theorem). The natural homomorphism $\oplus_{\lambda \in \sigma(A)} V(\lambda) \rightarrow V$ is bijective and, for each $\lambda \in \sigma(A)$, there exists a basis for $V(\lambda)$ such that each operator $A_{i}: V(\lambda) \rightarrow V(\lambda)$ is represented by an upper triangular matrix with $\lambda_{i}$ as the only diagonal entry. In particular, if $V(0) \neq\{0\}$, then it contains a common zero eigenvector for all $A_{i}$ 's.

As a consequence of the Lie theorem we get the next proposition.

Proposition 2.6. Suppose that $A$ is an $n$-tuple of commuting operators on a finite dimensional vector space $V$. Then

$$
H_{k}(A, V) \cong H_{k}(A, V(0))
$$

for all $k \in\{0, \ldots, n\}$. Furthermore, the homology group

$$
H_{n}(A, V(0)) \cong \cap_{i=1}^{n} \operatorname{Ker}\left(A_{i}\right)
$$

is nonzero if and only if $V(0) \neq\{0\}$.

Proof. Let $k \in\{0, \ldots, n\}$. By the above theorem, $H_{k}(A, V) \cong \oplus_{\lambda \in \sigma(A)} H_{k}(A, V(\lambda))$. Suppose now that $\lambda \in \sigma(A)$ and $\lambda \neq 0$, say $\lambda_{i} \neq 0$. The operator $A_{i}: V(\lambda) \rightarrow V(\lambda)$ is then invertible and the Koszul complex $K_{*}(A, V(\lambda))$ is therefore contractible. This proves the first part of the proposition.

The second part of the proposition follows from the bi-implications,

$$
V(0) \neq\{0\} \Leftrightarrow \cap_{i=1}^{n} \operatorname{Ker}\left(A_{i}\right) \neq\{0\} \Leftrightarrow H_{n}(A, V(0)) \neq\{0\} .
$$

The first one is part of the above theorem and the second one follows from the first part of the proposition since $H_{n}(A, V)=\cap_{i=1}^{n} \operatorname{Ker}\left(A_{i}\right)$.

\subsection{Taylor spectrum.}

From now on we will suppose that $V$ has the additional structure of a Banach space and that the commuting linear operators $A_{1}, \ldots, A_{n}: V \rightarrow V$ are bounded.

The Koszul complex can be used to define a good notion of joint spectrum of the n-tuple $A$, often referred to as the Taylor spectrum. See [20].

Definition 2.7. The (Taylor) spectrum of $A$ is the set

$$
\operatorname{Sp}(A):=\left\{\lambda \in \mathbb{C}^{n} \mid H_{*}(A-\lambda, V) \neq\{0\}\right\}
$$

where $A-\lambda:=\left(A_{1}-\lambda_{1}, \ldots, A_{n}-\lambda_{n}\right)$. The Taylor spectrum is a compact nonempty subset of $\mathbb{C}^{n},[20$, Theorem 3.1]. 
As an immediate corollary of Proposition 2.6 we get the following.

Corollary 2.8. Suppose that $V$ is finite dimensional. Then the following are equivalent:

(1) $\lambda \in \operatorname{Sp}(A)$

(2) $\lambda \in \sigma(A)$

(3) $H_{n}(A-\lambda, V) \neq\{0\}$.

The main result about the Taylor spectrum is the existence of a holomorphic functional calculus. In order to formulate this result we need to introduce some notation.

Notation 2.9. Let $\mathcal{O}(\operatorname{Sp}(A))$ denote the ring of germs of holomorphic functions on $\operatorname{Sp}(A)$, i.e. holomorphic functions $f: U \rightarrow \mathbb{C}$ defined on some open set $U \supseteq \operatorname{Sp}(A)$ subject to the equivalence relation:

$f: U \rightarrow \mathbb{C}$ and $g: V \rightarrow \mathbb{C}$ represent the same class in $\mathcal{O}(\operatorname{Sp}(A))$ if they agree on some open set $W$ with $\operatorname{Sp}(A) \subseteq W \subseteq U \cap V$.

The ring structure is given by the pointwise sum and product.

The notation $\mathcal{O}(U)$ refers to the unital ring of holomorphic functions on some domain $U \subseteq \mathbb{C}^{n}$.

For any subalgebra $\mathscr{B} \subseteq \mathscr{L}(V)$ of the bounded operators on $V$ its commutant is the algebra

$$
\mathscr{B}^{\prime}=\{C \in \mathscr{L}(V) \mid[C, B]=0, \text { for all } B \in \mathscr{B}\}
$$

Theorem 2.10. [19, Theorem 4.8] Let $\mathscr{A} \subseteq \mathscr{L}(V)$ denote the smallest unital $\mathbb{C}$ algebra which contains the bounded operators $A_{1}, \ldots, A_{n}$. There exists a unital homomorphism $\mathcal{O}(\operatorname{Sp}(A)) \rightarrow \mathscr{A}^{\prime \prime}, f \mapsto f(A)$ such that $z_{i} \mapsto A_{i}$. Furthermore, whenever $f=\left(f_{1}, \ldots, f_{m}\right): \operatorname{Sp}(A) \rightarrow \mathbb{C}^{m}$ is analytic $\left(i\right.$. e. $f_{k} \in \mathcal{O}(\operatorname{Sp}(A)), k=$ $1, \ldots, m)$ the following identity holds:

$$
\operatorname{Sp}(f(A))=f(\operatorname{Sp}(A))
$$

A uniqueness result for the holomorphic functional calculus is contained in the following.

Theorem 2.11. [4, Theorem 5.2.4] Let $U \supseteq \operatorname{Sp}(A)$ be an open set and let $\Phi: \mathcal{O}(U) \rightarrow \mathscr{L}(V)$ be a unital homomorphism. Suppose that $\Phi(f)=f(A)$ for all $f \in \mathcal{O}\left(\mathbb{C}^{n}\right)$ and that $h(\operatorname{Sp}(A))=\operatorname{Sp}(\Phi(h))$ for each holomorphic function $h: U \rightarrow \mathbb{C}^{l}, l \geq 1$. We then have the identity $\Phi(h)=h(A)$ for all $h \in \mathcal{O}(U)$. 
The holomorphic functional calculus is functorial with respect to $V$, in fact the following holds.

Proposition 2.12. [19, Proposition 4.5] Let $V$ and $W$ be Banach spaces, $\Phi: V \rightarrow$ $W$ be a bounded operator. Suppose that $A$ (resp. B) are commuting $n$-tuples on $V$ (resp. W) satisfying $B_{i} \Phi=\Phi A_{i}, i=1, \ldots n$. Then

$$
\Phi f(A)=f(B) \Phi .
$$

for all $f \in \mathcal{O}(\operatorname{Sp}(A) \cup \operatorname{Sp}(B))$.

Definition 2.13. In the context of the Theorem 2.10 we will endow $V$ with the structure of a module over $\mathcal{O}(\operatorname{Sp}(A))$ induced by the homomorphism $f \mapsto f(A)$.

For the rest of the section, suppose that $V$ is a Hilbert space over $\mathbb{C}$.

Let $\mathscr{C}(V)=\mathscr{L}(V) / \mathscr{K}(V)$ be the Calkin algebra, the quotient of the $\mathrm{C}^{*}$-algebra of bounded operators on $V$ by the ideal of compact operators. The commuting tuple $\pi(A)$ of bounded operators on $\mathscr{C}(V)$ is given by the action of the $A_{i}$ 's by left multiplication.

Definition 2.14. The essential spectrum of the commuting tuple $A$ is the Taylor spectrum of $\pi(A)$. It will be denoted by $\operatorname{Sp}_{\text {ess }}(A)$.

The relation between the Fredholmness of $A$ and the essential spectrum of $A$ was clarified by Curto in [2]. We state the result as a theorem.

Theorem 2.15. [2, Corollary 6.2] Let $\lambda \in \mathbb{C}^{n}$. The commuting tuple $A-\lambda$ is Fredholm if and only if $\lambda \notin \operatorname{Sp}_{\text {ess }}(A)$.

Let $g=\left(g_{1}, \ldots, g_{m}\right): \operatorname{Sp}(A) \rightarrow \mathbb{C}^{m}$ be an analytic map. In analogy with Theorem 2.10 we can characterize the Fredholmness of $g(A)$ in terms of the image set $g\left(\operatorname{Sp}_{\text {ess }}(A)\right) \subseteq \mathbb{C}^{m}$. See [10] and [5].

Proposition 2.16. The commuting tuple $g(A)$ is Fredholm if and only if $0 \notin$ $g\left(\operatorname{Sp}_{\text {ess }}(A)\right)$.

2.4. A spectral sequence. A generalization of the mapping cone observation in Lemma 2.4 is as follows.

Let $A=\left(A_{1}, \ldots, A_{n}\right)$ and $B=\left(B_{1}, \ldots, B_{m}\right)$ be commuting tuples of linear operators on a vector space $V$ such that the union $A \oplus B:=\left(A_{1}, \ldots, A_{n}, B_{1}, \ldots, B_{m}\right)$ is a commuting $(n+m)$-tuple.

Let us define a bigrading of the exterior algebra $\Lambda\left(\mathbb{C}^{n+m}\right)$ which reflects the position of $A$ and $B$ in the union $A \oplus B$. For each pair $p \in\{0,1, \ldots, n\}$ and $q \in\{0,1, \ldots, m\}$ we define the subspace

$$
\begin{aligned}
& \Lambda_{p, q}\left(\mathbb{C}^{n+m}\right) \\
& \quad:=\operatorname{span}\left\{e_{I} \wedge e_{J}|I \subseteq\{1, \ldots, n\},| I|=p, J \subseteq\{n+1, \ldots, n+m\},| J \mid=q\right\},
\end{aligned}
$$


where we recall that $e_{I}:=e_{i_{1}} \wedge \ldots \wedge e_{i_{k}}$ for any subset $I=\left\{i_{1}, \ldots, i_{k}\right\} \subseteq$ $\{1, \ldots, n+m\}$ with $i_{1}<\ldots<i_{k}$. We then have an isomorphism $\Lambda\left(\mathbb{C}^{n+m}\right) \cong$ $\oplus_{p=0}^{n} \oplus_{q=0}^{m} \Lambda_{p, q}\left(\mathbb{C}^{n+m}\right)$ and this decomposition turns $\Lambda\left(\mathbb{C}^{n+m}\right)$ into a bigraded algebra.

The bigrading of the exterior algebra leads to a bigrading of the Koszul chains $K_{*}(A \oplus B, V)=V \otimes \Lambda\left(\mathbb{C}^{n+m}\right)$ by defining $K_{p, q}(A \oplus B, V):=V \otimes$ $\Lambda_{p, q}\left(\mathbb{C}^{n+m}\right)$. This allows us to view the Koszul complex as the totalization of the following bicomplex. We define the vertical differential $d^{v}: K_{p, q}(A \oplus B, V) \rightarrow$ $K_{p-1, q}(A \oplus B, V)$ by $d^{v}:=\sum_{i=1}^{n} A_{i} \otimes \varepsilon_{i}^{*}$ and the horizontal differential by $d^{h}: K_{p, q}(A \oplus B, V) \rightarrow K_{p, q-1}(A \oplus B, V), d^{h}:=\sum_{i=1}^{m} B_{i} \otimes \varepsilon_{i+n}^{*}$. Since $\left(d^{v}\right)^{2}=\left(d^{h}\right)^{2}=d^{v} d^{h}+d^{h} d^{v}=0$ we get a bicomplex

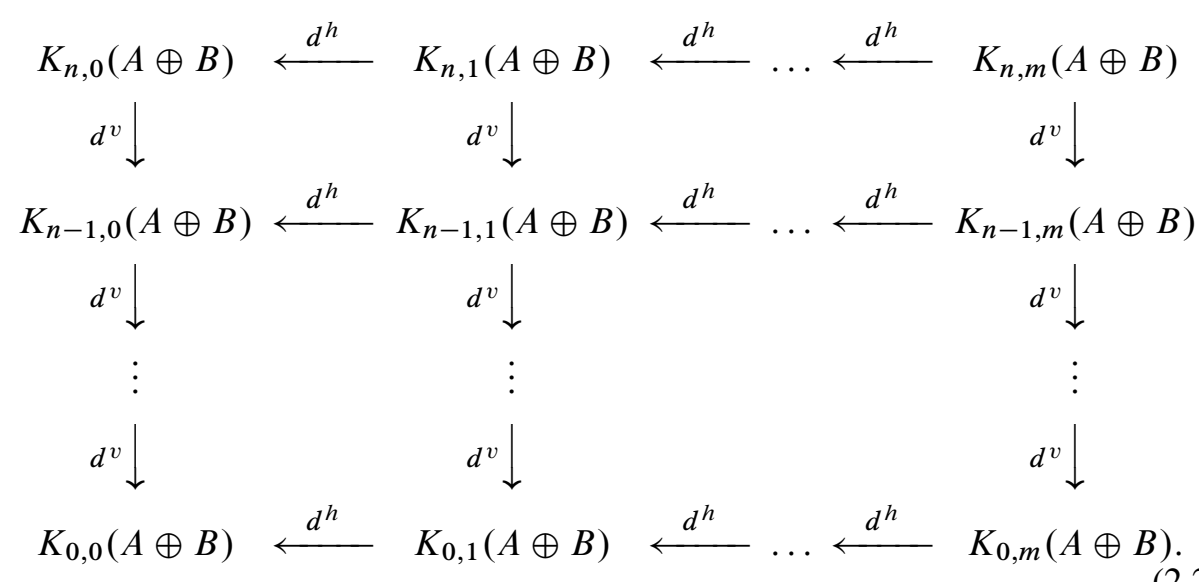

It is not hard to see that the totalization of this bicomplex is isomorphic to our original Koszul complex $K_{*}(A \oplus B, V)$.

Below we will describe the homology spectral sequence associated with the filtration by rows. For each $i \in\{0, \ldots, n\}$ we define the sub-bicomplex $F_{i}$ consisting of the rows with indices $0, \ldots, i$ of the bicomplex (2.2). This gives a filtration

$$
0 \subseteq F_{0} \subseteq F_{1} \subseteq \ldots \subseteq F_{n-1} \subseteq F_{n}=K_{* *}(A \oplus B, V)
$$

with an associated spectral sequence converging to the Koszul homology of $A \oplus B$ (see f. ex. [21, Theorem 5.5.1]).

Proposition 2.17. The homology spectral sequence associated with the row filtration of the bicomplex $K_{* *}(A \oplus B, V)$ converges to the Koszul homology of $A \oplus B$. The $E^{2}$-term of this spectral sequence is given by $E_{p q}^{2}=H_{p}\left(A, H_{q}(B, V)\right)$.

Proof. Let $p \in\{0, \ldots, n\}$ and $q \in\{0, \ldots, m\}$. By definition the $E_{p q}^{1}$-term is given by the $q^{\text {th }}$ homology group of the chain complex $F_{p} / F_{p-1}$. The chain complex $F_{p} / F_{p-1}$ is given by the $p^{\text {th }}$ row of the bicomplex $K_{* *}(A \oplus B, V)$ which is 
isomorphic to the chain-complex $\left(K_{*}(B, V) \otimes \Lambda_{p}\left(\mathbb{C}^{n}\right),(-1)^{p} \cdot d_{B} \otimes 1\right)$. The term $E_{p q}^{1}$ of our spectral sequence is thus given by $H_{q}(B, V) \otimes \Lambda_{p}\left(\mathbb{C}^{n}\right)$. The differential $d^{1}: E_{p q}^{1} \rightarrow E_{p-1, q}^{1}$ is nothing but the Koszul-differential of the commuting tuple $H_{q}(A):=\left(H_{q}\left(A_{1}\right), \ldots, H_{q}\left(A_{n}\right)\right)$ which acts on the homology group $H_{q}(B, V)$. It follows that the $E_{p q}^{2}$-term is given by the homology group $H_{p}\left(A, H_{q}(B, V)\right)$ as desired.

In the remainder of this section we will prove corollaries which will be needed later on. The first two explore the link between the invertibility of the union $A \oplus B$ and the invertibility of the individual terms $A$ and $B$.

Proposition 2.18. Suppose that $B=\left(B_{1}, \ldots, B_{m}\right)$ is Fredholm and that the Koszul homology group $H_{p}\left(A, H_{q}(B, V)\right)$ is non-trivial for some $p \in\{0, \ldots, n\}$ and $q \in\{0, \ldots, m\}$. Then there exists $a k \geq p+q$ such that $H_{k}(A \oplus B, V)$ is nontrivial as well.

Proof. Let $\left(E^{r}, d^{r}\right)$ denote the homology spectral sequence associated with the row filtration of the bicomplex $K_{* *}(A \oplus B, V)$. Recall that the differential $d^{r}$ sends $E_{p q}^{r}$ to $E_{p-r, q+r-1}^{r}$.

Since $B$ is Fredholm by assumption, $H_{q}(B, V)$ is finite dimensional. By Corollary 2.8 the non-triviality of the homology group $H_{p}\left(A, H_{q}(B, V)\right)$ implies that the homology group $H_{n}\left(A, H_{q}(B, V)\right)$ is non-trivial as well.

To continue, note that the homology group $E_{n q}^{r+1}$ can be identified with the kernel of the differential $d^{r}: E_{n q}^{r} \rightarrow E_{n-r, q+r-1}^{r}$ for all $r \in \mathbb{N}$. By Proposition 2.17 $H_{n}\left(A, H_{q}(B, V)\right) \cong E_{n q}^{2}$. The above reasoning therefore gives us a non-trivial vector $\xi \in E_{n q}^{2}$. Suppose now that $\xi \in E_{n q}^{2}$ determines a class in $E_{n q}^{r}$ for all $r \geq 2$ thus that $d^{r}(\xi)=0$ for all $r \geq 2$. This implies that the limit $E_{n q}^{\infty}$ is non-trivial and hence, by the convergence of the spectral sequence, that the homology group $H_{n+q}(A \oplus B, V)$ is non-trivial. Since $n+q \geq p+q$ we can thus assume, without loss of generality, the existence of an $r_{0} \geq 2$, such that $\xi$ determines a class in $E_{n q}^{r_{0}}$ but $d^{r_{0}}(\xi) \neq 0$.

This assumption implies in particular that the homology group $E_{n-r_{0}, q+r_{0}-1}^{r_{0}}$ is non-trivial. But this can only happen if the homology group $E_{n-r_{0}, q+r_{0}-1}^{2}=$ $H_{n-r_{0}}\left(A, H_{q+r_{0}-1}(B, V)\right)$ is non-trivial as well. By the Fredholmness assumption on $B$ the homology group $H_{n}\left(A, H_{j}(B, V)\right), j=q+r_{0}-1>q$, is non-zero. Applying the same argument a finite number of times we may assume, without loss of generality, that the homology group $H_{n}\left(A, H_{m}(B, V)\right)$ is non-trivial. But this group agrees with the $E_{n m}^{\infty}$-term of the spectral sequence since $d^{r}: E_{n m}^{r} \rightarrow$ $E_{n-r, m+r-1}^{r}$ is the trivial map for all $r \geq 2$. By the convergence of the spectral sequence, this implies that $H_{n+m}(A \oplus B, V)$ is non-trivial and the claim of the proposition is proved. 
Proposition 2.19. Let $k \in\{0, \ldots, n+m\}$ and suppose that the homology group $H_{p}\left(A, H_{q}(B, V)\right)$ is trivial for all $p \in\{0, \ldots, n\}$ and all $q \in\{0, \ldots, m\}$ with $p+q=k$. Then the homology group $H_{k}(A \oplus B, V)$ is trivial.

Proof. Let $\left(E^{r}, d^{r}\right)$ denote as above the homology spectral sequence associated with the row filtration of the bicomplex $K_{* *}(A \oplus B, V)$. By Proposition 2.17 the $E^{2}$-term is given by $E_{p q}^{2}:=H_{p}\left(A, H_{q}(B, V)\right)$. The assumptions imply that $E_{p q}^{2}=0$ for all $p, q$ with $p+q=k$. The convergence of the spectral sequence entails that $H_{k}(A \oplus B, V)$ is trivial as well.

The last proposition states the expected relation between the index of the union $A \oplus B$ and the indices of the invidual terms $A$ and $B$. This result is not particular for the present situation and follows from general facts about spectral sequences. We have included a full proof here for the sake of completeness. It is important to note that the assumption of Fredholmness of the commuting tuple $B$ is too strict in general since it implies that the index of the union $A \oplus B$ is trivial. It is in particular unrealistic to assume that the chains in the bicomplex $K_{* *}(A \oplus B)$ are finite dimensional.

Proposition 2.20. Suppose that the homology group $H_{p}\left(A, H_{q}(B, V)\right)$ is finite dimensional for all $p \in\{0, \ldots, n\}$ and $q \in\{0, \ldots, m\}$. Then the commuting tuple $A \oplus B$ is Fredholm and the index is given by

$$
\operatorname{Ind}(A \oplus B)=\sum_{p, q}(-1)^{p+q+1} \operatorname{Dim}\left(H_{p}\left(A, H_{q}(B, V)\right)\right) .
$$

Proof. By an application of Proposition 2.17 we see that the $E^{2}$-term of the homology spectral sequence associated with the row filtration of $K_{* *}(A \oplus B, V)$ is finite dimensional. Since each term of the spectral sequence is obtained by taking homology groups of its predecessor we get that the $E^{r}$-term is finite dimensional for all $r \geq 2$. The convergence of the spectral sequence then implies that $\operatorname{Dim}_{\mathbb{C}}\left(H_{k}(A \oplus B, V)\right)=\sum_{p+q=k} \operatorname{Dim}_{\mathbb{C}}\left(E_{p q}^{\infty}\right)$ is finite for each $k \in\{0, \ldots, n+m\}$. This means that $A \oplus B$ is Fredholm. Furthermore, we see that

$$
\operatorname{Ind}(A \oplus B)=\sum_{p, q}(-1)^{p+q+1} \operatorname{Dim}_{\mathbb{C}}\left(E_{p q}^{\infty}\right)
$$

Recall now that for a chain complex of finite dimensional vector spaces, the alternating sum of dimensions of the chains agrees with the alternating sum of dimensions of the homology groups. Using this fact together with the observation that each term of the spectral sequence is the homology of its predecessor we get that

$$
\sum_{p, q}(-1)^{p+q+1} \operatorname{Dim}_{\mathbb{C}}\left(E_{p q}^{\infty}\right)=\sum_{p, q}(-1)^{p+q+1} \operatorname{Dim}_{\mathbb{C}}\left(E_{p q}^{r}\right)
$$

for all $r \geq 2$. This proves the claim of the proposition since $E_{p q}^{2}=H_{p}\left(A, H_{q}(B, V)\right)$. 


\section{Spectrum of Fredholm tuples}

Notation 3.1. Let $A=\left(A_{1}, \ldots, A_{n}\right)$ be a commuting tuple of bounded operators on a Hilbert space $\mathscr{H}$, and let $g: \mathrm{Sp}(A) \rightarrow \mathbb{C}^{m}$ be a holomorphic map.

- $g(A)=\left(g_{1}(A), \ldots, g_{m}(A)\right)$ denotes the commuting tuple obtained from $A$ and $g$ by the holomorphic functional calculus.

- Since each of the operators $g_{j}(A)$ commute with each of the operators $A_{i}$, the $A_{i}$ 's induce a commuting tuple of linear operators on the Koszul homology groups $H_{*}(g(A), \mathscr{H})$. We will denote this n-tuple by

$$
H_{*}(A):=\left(H_{*}\left(A_{1}\right), \ldots, H_{*}\left(A_{n}\right)\right) .
$$

- $Z(g):=\left\{\lambda \in \operatorname{Sp}(A) \mid g_{1}(\lambda)=\ldots=g_{m}(\lambda)=0\right\}$ is the set of common zeroes.

When $g(A)$ is Fredholm, the Taylor spectum $\operatorname{Sp}\left(H_{k}(A)\right)$ makes sense and is a finite set for each $k \in\{0, \ldots, m\}$. In this case let

$$
\operatorname{Sp}(H(A)):=\cup_{k=0}^{m} \operatorname{Sp}\left(H_{k}(A)\right) .
$$

Lemma 3.2. The set of common zeroes $Z(g)$ agrees with the set $\left\{\lambda \in \mathbb{C}^{n} \mid(\lambda, 0) \in\right.$ $\mathrm{Sp}(A \oplus g(A))\}$.

Proof. In fact, by Theorem 2.10, $\operatorname{Sp}(A \oplus g(A))$ coincides with the graph of the map $g: \operatorname{Sp}(A) \rightarrow \mathbb{C}^{m}$.

Theorem 3.3. Suppose that $g(A)$ is Fredholm. Then

$$
Z(g)=\operatorname{Sp}(H(A)) .
$$

In particular, the set of common zeroes for $g$ is finite.

Proof. The result of the theorem follows from the following bi-implications:

$$
\begin{aligned}
\lambda \in Z(g) & \Leftrightarrow H_{*}((A-\lambda) \oplus g(A), \mathscr{H}) \neq\{0\} \Leftrightarrow H_{*}\left(A-\lambda, H_{*}(g(A), \mathscr{H})\right) \neq\{0\} \\
& \Leftrightarrow \lambda \in \operatorname{Sp}(H(A)) .
\end{aligned}
$$

The first bi-implication is the statement of Lemma 3.2 and the second one follows from Proposition 2.18 and Proposition 2.19. The last bi-implication follows by definition of the set $\operatorname{Sp}(H(A))$.

Notation 3.4. For each common zero $\lambda \in Z(g)$ and each $k \in\{0, \ldots, m\}$, $H_{k}(g(A), \mathscr{H})(\lambda) \subseteq H_{k}(g(A), \mathscr{H})$ denotes the finite dimensional vector space coming from the spectral decomposition of $H_{k}(g(A), \mathscr{H})$ with respect to the commuting tuple $H_{k}(A)$ (see Section 2.2). 
The above spectral decomposition of the Koszul homology groups allows us to define a local version of the Fredholm index.

Definition 3.5. Suppose that $g(A)$ is Fredholm and that $\lambda \in Z(g)$. The local index of $g(A)$ at $\lambda$ is the integer

$$
\operatorname{Ind}_{\lambda}(g(A)):=\sum_{k=0}^{m}(-1)^{k+1} \operatorname{Dim}_{\mathbb{C}}\left(H_{k}(g(A), \mathscr{H})(\lambda)\right) .
$$

The relation between the local indices for $g(A)$ and the global index for $g(A)$ is given by the following:

Proposition 3.6. Suppose that $g(A)$ is Fredholm. Then the index of $g(A)$ can be computed as the sum of the local indices. Thus,

$$
\operatorname{Ind}(g(A))=\sum_{\lambda \in Z(g)} \operatorname{Ind}_{\lambda}(g(A)) .
$$

Proof. Let $k \in\{0, \ldots, m\}$. By Theorem 2.5, Corollary 2.8, and Theorem 3.3 we have the following isomorphisms

$$
\begin{aligned}
H_{k}(g(A), \mathscr{H}) & \cong \bigoplus_{\lambda \in \sigma\left(H_{k}(A)\right)} H_{k}(g(A), \mathscr{H})(\lambda)=\bigoplus_{\lambda \in \operatorname{Sp}\left(H_{k}(A)\right)} H_{k}(g(A), \mathscr{H})(\lambda) \\
& \cong \bigoplus_{\lambda \in Z(g)} H_{k}(g(A), \mathscr{H})(\lambda)
\end{aligned}
$$

where we recall that $H_{k}(g(A), \mathscr{H})(\lambda)=\{0\}$ whenever $\lambda \notin \sigma\left(H_{k}(A)\right)$. This immediately implies the result of the proposition.

\section{Algebraic localization}

Let $\mathscr{H}$ be a Hilbert space and let $A=\left(A_{1}, \ldots, A_{n}\right)$ be a commuting tuple of bounded operators on $\mathscr{H}$. Recall that the holomorphic functional calculus (Theorem 2.10) gives $\mathscr{H}$ the structure of a $\mathcal{O}(\mathrm{Sp}(A))$-module.

Throughout this section the following assumption will be in use.

Let $g: \operatorname{Sp}(A) \rightarrow \mathbb{C}^{m}$ be a holomorphic map such that $g(A):=\left(g_{1}(A), \ldots, g_{m}(A)\right)$ is Fredholm. Let $\lambda \in \mathrm{Sp}(A)$.

By the results in Section 3 there is a decomposition

$$
H_{k}(g(A), \mathscr{H}) \cong \bigoplus_{\mu \in Z(g)} H_{k}(g(A), \mathscr{H})(\mu)
$$

for each $k \in\{0, \ldots, m\}$, where the subspaces $H_{k}(g(A), \mathscr{H})(\mu) \subseteq H_{k}(g(A), \mathscr{H})$ are generalized eigenspaces for the commuting tuple $H_{k}(A):=\left(H_{k}\left(A_{1}\right), \ldots, H_{k}\left(A_{n}\right)\right)$. 
Notation 4.1. For each $f \in \mathcal{O}(\operatorname{Sp}(A))$ and each $k \in 0, \ldots, m$, let

$$
H_{k}(f(A))(\lambda): H_{k}(g(A), \mathscr{H})(\lambda) \rightarrow H_{k}(g(A), \mathscr{H})(\lambda)
$$

denote the endomorphism of the generalized eigenspace for $H_{k}(A)$ at the point $\lambda \in \mathrm{Sp}(A)$ obtained by restriction of the endomorphism $H_{k}(f(A)): H_{k}(g(A), \mathscr{H}) \rightarrow$ $H_{k}(g(A), \mathscr{H})$. In particular we have the commuting tuple

$$
H_{k}(A)(\lambda):=\left(H_{k}\left(A_{1}\right)(\lambda), \ldots, H_{k}\left(A_{n}\right)(\lambda)\right) .
$$

Let $f \in \mathcal{O}(\operatorname{Sp}(A))$ and let $k \in\{0, \ldots, m\}$. Since $H\left(A_{i}\right)(\lambda)-\lambda_{i}$ is nilpotent for each $i \in\{1, \ldots, n\}$ we get that $\operatorname{Sp}\left(H_{k}(A)(\lambda)\right) \subseteq\{\lambda\}$. The holomorphic functional calculus therefore yields a linear operator

$$
f\left(H_{k}(A)(\lambda)\right): H_{k}(g(A), \mathscr{H})(\lambda) \rightarrow H_{k}(g(A), \mathscr{H})(\lambda) .
$$

Remark that it follows from the decomposition in (4.1) that $H_{k}(g(A), \mathscr{H})(\lambda)=\{0\}$ when $g(\lambda) \neq 0$. The only interesting case is thus when $g(\lambda)=0$.

The first aim of this section is to prove the identity

$$
f\left(H_{k}(A)(\lambda)\right)=H_{k}(f(A))(\lambda) .
$$

We start by proving this identity when $f$ is the restriction of a holomorphic map on $\mathbb{C}^{n}$.

Lemma 4.2. For each $f \in \mathcal{O}\left(\mathbb{C}^{n}\right)$ and each $k \in\{0, \ldots, m\}$ we have the identity

$$
f\left(H_{k}(A)(\lambda)\right)=H_{k}(f(A))(\lambda)
$$

of endomorphisms of the generalized eigenspace $H_{k}(g(A), \mathscr{H})(\lambda)$.

Proof. Let $Z_{k}$ denote the kernel of the differential $d_{g(A)}:=\sum_{l=1}^{m} g_{l}(A) \otimes \varepsilon_{l}^{*}$ : $\mathscr{H} \otimes \Lambda_{k}\left(\mathbb{C}^{m}\right) \rightarrow \mathscr{H} \otimes \Lambda_{k-1}\left(\mathbb{C}^{m}\right)$. Let $q: Z_{k} \rightarrow H_{k}(g(A), \mathscr{H})$ denote the quotient map and let $\iota: Z_{k} \rightarrow \mathscr{H} \otimes \Lambda_{k}\left(\mathbb{C}^{m}\right)$ denote the inclusion. Both of these linear maps are bounded and we have the identities $A_{i} \iota=\left.\iota A_{i}\right|_{Z_{k}}$ and $\left.q A_{i}\right|_{Z_{k}}=H_{k}\left(A_{i}\right) q$ for all $i \in\{1, \ldots, n\}$. Since $f \in \mathcal{O}\left(\mathbb{C}^{n}\right)$ we can conclude from Proposition 2.12 that

$$
f\left(H_{k}(A)\right)=H_{k}(f(A)) .
$$

Notice that the assumption $f \in \mathcal{O}\left(\mathbb{C}^{n}\right)$ is needed since we have no control over the spectrum of the commuting tuple $\left.A\right|_{Z_{k}}:=\left(A_{1}\left|Z_{k}, \ldots, A_{n}\right| Z_{k}\right)$.

This proves the claim of the lemma since

$$
\oplus_{\mu \in Z(g)} f\left(H_{k}(A)(\mu)\right)=f\left(H_{k}(A)\right)=H_{k}(f(A))=\oplus_{\mu \in Z(g)} H_{k}(f(A))(\mu),
$$

where an operator of the form $\oplus_{\mu \in Z(g)} T_{\mu}: H_{k}(g(A), \mathscr{H}) \rightarrow H_{k}(g(A), \mathscr{H})$ is induced by the block diagonal matrix with the linear operators $T_{\mu}: H_{k}(g(A), \mathscr{H})(\mu) \rightarrow$ $H_{k}(g(A), \mathscr{H})(\mu)$ on the diagonal. 
The next lemma allows us to compute the spectrum of endomorphisms of the form $H_{k}(f(A))(\lambda): H_{k}(g(A), \mathscr{H})(\lambda) \rightarrow H_{k}(g(A), \mathscr{H})(\lambda)$ without the assumption that $f \in \mathcal{O}\left(\mathbb{C}^{n}\right)$.

Lemma 4.3. Suppose that $f: \operatorname{Sp}(A) \rightarrow \mathbb{C}$ is holomorphic and $f(\lambda) \neq 0$. The endomorphism $H_{k}(f(A))(\lambda)$ is then invertible for all $k \in\{0, \ldots, m\}$.

Proof. The mapping cone construction of the Koszul complex (Lemma 2.4) yields a long exact sequence

$$
\begin{gathered}
\ldots \longrightarrow H_{k+1}(f \oplus g, \mathscr{H}) \longrightarrow H_{k}(g, \mathscr{H}) \stackrel{H_{k}(f)}{\longrightarrow} H_{k}(g, \mathscr{H}) \\
\ldots \\
\ldots H_{k-1}(f \oplus g, \mathscr{H}) \longleftarrow H_{k}(f \oplus g, \mathscr{H})
\end{gathered}
$$

of homology groups. It can be verified that each of the linear maps in this sequence intertwines the action of the commuting tuple $A$. They are in fact homorphisms for the $\mathcal{O}(\operatorname{Sp}(A))$-module structure on the involved homology groups. See [8] for an explicit description of these maps at the level of complexes. In particular we get a long exact sequence

$$
\begin{gathered}
\longrightarrow H_{k+1}(f \oplus g, \mathscr{H})(\mu) \longrightarrow H_{k}(g, \mathscr{H})(\mu) \stackrel{H_{k}(f)(\mu)}{\longrightarrow} H_{k}(g, \mathscr{H})(\mu) \\
\ldots \\
\ldots H_{k-1}(f \oplus g, \mathscr{H})(\mu) \stackrel{\downarrow}{\longleftarrow} H_{k}(f \oplus g, \mathscr{H})(\mu)
\end{gathered}
$$

for each $\mu \in \operatorname{Sp}(A)$. It is therefore enough to show that the generalized eigenspace $H_{*}(f(A) \oplus g(A), \mathscr{H})(\lambda)$ is trivial. This is a consequence of Theorem 3.3 since $\lambda \notin Z(f) \cap Z(g)$.

The uniqueness result for the holomorphic functional calculus (Theorem 2.11) now allows us to prove the identity in Equation (4.2).

Proposition 4.4. For each $f \in \mathcal{O}(\operatorname{Sp}(A))$ and each $k \in\{0, \ldots, m\}$ we have the identity

$$
f\left(H_{k}(A)(\lambda)\right)=H_{k}(f(A))(\lambda)
$$

of endomorphisms of the generalized eigenspace $H_{k}(g(A), \mathscr{H})(\lambda)$.

Proof. Let $k \in\{0, \ldots, m\}$ and let $\Phi: \mathcal{O}(\mathrm{Sp}(A)) \rightarrow \mathscr{L}\left(H_{k}(g(A), \mathscr{H})(\lambda)\right)$ denote the unital homomorphism $\Phi(f):=H_{k}(f(A))(\lambda)$. We need to show that $\Phi(f)=$ $f\left(H_{k}(A)(\lambda)\right)$ for all $f \in \mathcal{O}(\operatorname{Sp}(A))$.

By Theorem 2.11 we only need to prove that $\Phi(f)=f\left(H_{k}(A)(\lambda)\right)$ for all $f \in \mathcal{O}\left(\mathbb{C}^{n}\right)$ and that $h\left(\mathrm{Sp}\left(H_{k}(A)(\lambda)\right)\right)=\mathrm{Sp}(\Phi(h))$ for all holomorphic functions $h: \mathrm{Sp}(A) \rightarrow \mathbb{C}^{i}, i \geq 1$. 
The first of these assertions is the content of Lemma 4.2.

To verify the second assertion, let $h: \operatorname{Sp}(A) \rightarrow \mathbb{C}^{i}$ be a holomorphic function for some $i \geq 1$. Without loss of generality we may assume that $H_{k}(g(A), \mathscr{H})(\lambda) \neq\{0\}$. We then have that $\operatorname{Sp}\left(H_{k}(A)(\lambda)\right)=\{\lambda\}$ and the desired identity becomes $h(\lambda)=\operatorname{Sp}(\Phi(h))$. Since the Taylor spectrum of $\Phi(h)$ is nonempty we only need to show that $\operatorname{Sp}(\Phi(h)) \subseteq\{h(\lambda)\}$ or in other words that $\Phi(h)-\mu$ is invertible whenever $\mu \neq h(\lambda)$. But this is an easy consequence of Lemma 4.3.

Notation 4.5. For each $\lambda \in \mathbb{C}^{n}$, let $\mathcal{O}_{\lambda}$ denote the stalk of the sheaf of analytic functions on $\mathbb{C}^{n}$ at $\lambda$. This stalk can be identified with the unital commutative ring of convergent power series near $\lambda$. When $\lambda \in \operatorname{Sp}(A)$ there is a well-defined restriction $\operatorname{map} \mathcal{O}(\operatorname{Sp}(A)) \rightarrow \mathcal{O}_{\lambda}$.

As an application of the above result we have the following useful:

Proposition 4.6. Suppose that $g(A)=\left(g_{1}(A), \ldots, g_{m}(A)\right)$ is Fredholm. Let $\lambda \in$ $\operatorname{Sp}(A)$ and let $k \in\{0, \ldots, m\}$. Then the generalized eigenspace $H_{k}(g(A), \mathscr{H})(\lambda)$ can be turned into a module over the ring $\mathcal{O}_{\lambda}$ of convergent power series in such a way that the associated homomorphism $\mathcal{O}_{\lambda} \rightarrow \mathscr{L}\left(H_{k}(g(A), \mathscr{H})(\lambda)\right)$ makes the diagram

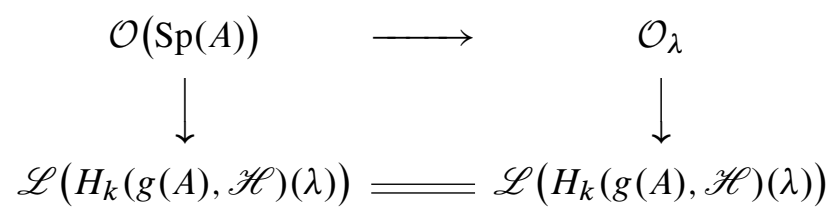

commute. Here the upper horizontal map is the restriction homomorphism and the left vertical map is the homomorphism associated with the action of $\mathcal{O}(\operatorname{Sp}(A))$ on $H_{k}(g(A), \mathscr{H})(\lambda)$.

Proof. The existence of a unital graded homomorphism $\mathcal{O}_{\lambda} \rightarrow \mathscr{L}\left(H_{k}(g(A), \mathscr{H})(\lambda)\right)$ which maps the coordinates $z_{i}-\lambda_{i}$ to $H_{k}\left(A_{i}-\lambda_{i}\right)(\lambda)$ is a consequence of the holomorphic functional calculus. Indeed, we have that $\operatorname{Sp}\left(H_{k}(A)(\lambda)\right) \subseteq\{\lambda\}$.

In order to prove the lemma we therefore only need to verify the identity $H_{k}(f(A))(\lambda)=f\left(H_{k}(A)(\lambda)\right)$ for each $f \in \mathcal{O}(\operatorname{Sp}(A))$. But this is the content of Proposition 4.4.

Let $\mathcal{M}$ be a left module over a unital commutative ring $\mathcal{B}$. Recall that the localization of $\mathcal{M}$ with respect to a prime ideal $\mathfrak{p} \subseteq \mathcal{B}$ consists of equivalence classes $\xi / s$, where $\xi \in \mathcal{M}$ and $s \notin \mathfrak{p}$. The equivalence classes are subject to the relation

$$
\xi / s=\eta / t \Leftrightarrow(\exists r \notin \mathfrak{p}:(r t) \cdot \xi=(r s) \cdot \eta) .
$$

The localization remains a left module over $\mathcal{B}$ with left action defined by $b \cdot(\xi / s):=$ $(b \xi) / s$. 
Notation 4.7. For each $\lambda \in \operatorname{Sp}(A)$, let $\mathscr{H}_{\lambda}$ denote the localization of the module $\mathscr{H}$ with respect to the prime ideal $\mathfrak{p}_{\lambda}:=\{f \in \mathcal{O}(\operatorname{Sp}(A)) \mid f(\lambda)=0\} \subseteq \mathcal{O}(\operatorname{Sp}(A))$.

Our results allow us to describe the generalized eigenspace $H_{*}(g(A), \mathscr{H})(\lambda)$ using the Koszul homology groups $H_{*}\left(g, \mathscr{H}_{\lambda}\right)$.

Proposition 4.8. Suppose that $g(A)$ is Fredholm. Let $\lambda \in \operatorname{Sp}(A)$ and let $k \in$ $\{0, \ldots, m\}$. Then there exists an isomorphism $H_{k}\left(g, \mathscr{H}_{\lambda}\right) \cong H_{k}(g(A), \mathscr{H})(\lambda)$ of modules over $\mathcal{O}(\operatorname{Sp}(A))$.

Proof. We start by noting that $H_{k}\left(g, \mathscr{H}_{\lambda}\right)$ is isomorphic to $H_{k}(g(A), \mathscr{H})_{\lambda}$ where as above $H_{k}(g(A), \mathscr{H})_{\lambda}$ denotes the localization of the module $H_{k}(g(A), \mathscr{H})$ with respect to the prime ideal $\mathfrak{p}_{\lambda} \subseteq \mathcal{O}(\operatorname{Sp}(A))$. This is a standard result about localizations, see for example [16, Chapter IV, §2].

We thus only need to prove that $H_{k}(g(A), \mathscr{H})_{\lambda} \cong H_{k}(g(A), \mathscr{H})(\lambda)$.

Let $f: \operatorname{Sp}(A) \rightarrow \mathbb{C}$ be holomorphic with $f \notin \mathfrak{p}_{\lambda}$. Since $f(\lambda) \neq 0$ we have that the image of $f$ in $\mathcal{O}_{\lambda}$ is invertible. It therefore follows from Proposition 4.6 that the induced homomorphism $f: H_{k}(g(A), \mathscr{H})(\lambda) \rightarrow H_{k}(g(A), \mathscr{H})(\lambda)$ is invertible.

Let $E_{\lambda}: H_{k}(g(A), \mathscr{H}) \rightarrow H_{k}(g(A), \mathscr{H})$ denote the projection onto $H_{k}(g(A)$, $\mathscr{H})(\lambda)$ relative to the decomposition $\oplus_{\lambda \in \mathrm{Sp}(A)} H_{k}(g(A), \mathscr{H})(\lambda) \cong H_{k}(g(A), \mathscr{H})$.

We then have a well-defined homomorphism of modules $H_{k}(g(A), \mathscr{H})_{\lambda} \rightarrow$ $H_{k}(f(A), \mathscr{H})(\lambda)$ defined by $\xi / f \mapsto f^{-1}\left(E_{\lambda} \xi\right)$. We claim that this homomorphism is an isomorphism with inverse given by $\xi \mapsto \xi / 1$. To prove this claim we mainly need to show that $\left(E_{\lambda} \xi\right) / 1=\xi / 1$ in $H_{k}(g(A), \mathscr{H})_{\lambda}$. Or in other words, we need to prove that $\xi / 1=0$ whenever $\xi \in H_{k}(g(A), \mathscr{H})(\mu)$ for some $\mu \neq \lambda$. However, for each such $\xi$ we can find a polynomial $p \in \mathcal{O}(\operatorname{Sp}(A)) \backslash \mathfrak{p}_{\lambda}$ such that $p \cdot \xi=0$. Indeed, we could choose $p=\left(z_{i}-\mu_{i}\right)^{l}$ where $\mu_{i} \neq \lambda_{i}$ and $l \in \mathbb{N}$ is large. But this implies that $\xi / 1=0$ as desired.

\section{The local index theorem for regular zeroes}

In this section the following general assumption will be in effect.

Let $g: \operatorname{Sp}(A) \rightarrow \mathbb{C}^{m}$ be a holomorphic map on the Taylor spectrum of our commuting tuple $A=\left(A_{1}, \ldots, A_{n}\right)$ with $m \geq n$. Suppose that $g(A)$ is Fredholm and that $\lambda \in Z(g)$ is a common zero which is also a regular point for the first $n$ coordinates $\left(g_{1}, \ldots, g_{n}\right): \operatorname{Sp}(A) \rightarrow \mathbb{C}^{n}$.

The aim is now to compute the local index of $g(A)$ at the regular point $\lambda \in Z(g)$. We shall see that it agrees with $\operatorname{Ind}(A-\lambda)$. On our way we obtain an even stronger result which expresses the dimensions of the localized homology groups $H_{*}(g(A), \mathscr{H})(\lambda)$ in terms of the dimensions of the homology groups $H_{*}(A-$ $\lambda, \mathscr{H})$. The regularity assumption on $\lambda \in Z(g)$ is crucial for obtaining this kind of expressions. 
Let $p_{n}(g):=\left(g_{1}, \ldots, g_{n}\right)$ and recall that $\lambda$ is said to be a regular point for $p_{n}(g)$ when the determinant of the Jacobian matrix $D\left(g_{1}, \ldots, g_{n}\right)=\operatorname{det}\left(\frac{\partial g_{i}}{\partial z_{j}}\right)$ is nontrivial at $\lambda$. Most importantly for our purposes this implies that the homomorphism $\mathcal{O}_{0} \rightarrow \mathcal{O}_{\lambda}, f \mapsto f \circ p_{n}(g)$ is an isomorphism of local rings. In particular we get the identity $\mathfrak{m}_{\lambda}=g_{1} \mathcal{O}_{\lambda}+\ldots+g_{n} \mathcal{O}_{\lambda}$ where $\mathfrak{m}_{\lambda}$ denotes the maximal ideal in $\mathcal{O}_{\lambda}$. See [9, Theorem 1.19].

As in Section 4, the notation $\mathscr{H}_{\lambda}$ refers to the localization of the module $\mathscr{H}$ with respect to the prime ideal $\mathfrak{p}_{\lambda} \subseteq \mathcal{O}(\operatorname{Sp}(A))$ of holomorphic functions which vanish at $\lambda$.

In the next lemmas we prove various triviality results for actions on Koszul homology groups.

Lemma 5.1. Let $B=\left(B_{1}, \ldots, B_{k}\right)$ be a commuting tuple such that the union $A \oplus$ $B=\left(A_{1}, \ldots, A_{n}, B_{1}, \ldots, B_{k}\right)$ commutes as well. Let $f \in \mathcal{O}(\mathrm{Sp}(A))$ and suppose that $f(\mu)=0$ for some $\mu \in \operatorname{Sp}(A)$. Then the endomorphism $H_{*}(f(A)): H_{*}((A-$ $\mu) \oplus B, \mathscr{H}) \rightarrow H_{*}((A-\mu) \oplus B, \mathscr{H})$ is trivial.

Proof. Let $p_{n}: \mathbb{C}^{n+k} \rightarrow \mathbb{C}^{n}$ denote the projection onto the first $n$ coordinates. Then $f(A)=\left(f \circ p_{n}\right)(A \oplus B)$. See f. ex. [4, Theorem 5.2.3].

It now follows from [19, Proposition 4.6] that the induced endomorphism

$H_{*}(f(A))=H_{*}\left(\left(f \circ p_{n}\right)(A \oplus B)\right): H_{*}((A-\mu) \oplus B, \mathscr{H}) \rightarrow H_{*}((A-\mu) \oplus B, \mathscr{H})$

is given by the scalar multiplication with $\left(f \circ p_{n}\right)(\mu, 0)=0$. This proves the lemma.

Lemma 5.2. Suppose that $\lambda \in \operatorname{Sp}(A)$ is a regular point for $p_{n}(g)=\left(g_{1}, \ldots, g_{n}\right)$ : $\operatorname{Sp}(A) \rightarrow \mathbb{C}^{n}$. Then the endomorphism

$$
H_{*}\left(z_{i}-\lambda_{i}\right): H_{*}\left(g \oplus h, \mathscr{H}_{\lambda}\right) \rightarrow H_{*}\left(g \oplus h, \mathscr{H}_{\lambda}\right)
$$

is trivial for each $i \in\{1, \ldots, n\}$ and each holomorphic function $h: \operatorname{Sp}(A) \rightarrow \mathbb{C}^{k}$, $k \geq 0($ for $k=0$ we let $g \oplus h:=g)$.

Proof. Let $i \in\{1, \ldots, n\}$ and let $h: \operatorname{Sp}(A) \rightarrow \mathbb{C}^{k}$ be holomorphic.

By Proposition 4.6 and Proposition 4.8 the action of $\mathcal{O}(\mathrm{Sp}(A))$ on $H_{*}(g \oplus h$, $\mathscr{H}_{\lambda}$ ) factorizes through the stalk $\mathcal{O}_{\lambda}$. It follows furthermore from the regularity of $p_{n}(g)$ at the point $\lambda \in \operatorname{Sp}(A)$ that the maximal ideal $\mathfrak{m}_{\lambda}$ is given by $g_{1} \mathcal{O}_{\lambda}+\ldots+$ $g_{n} \mathcal{O}_{\lambda}$. Since $z_{i}-\lambda_{i}$ determines an element in $\mathfrak{m}_{\lambda}$ it is enough to show that the endomorphism $H_{*}\left(g_{j}\right): H_{*}\left(g \oplus h, \mathscr{H}_{\lambda}\right) \rightarrow H_{*}\left(g \oplus h, \mathscr{H}_{\lambda}\right)$ is trivial for each $j \in\{1, \ldots, n\}$. But this is a well-known property of the Koszul homology groups, see [16, Chapter IV, Proposition 4].

The following lemma allows us to apply our two triviality results to compute the dimensions of certain Koszul homology groups. 
Lemma 5.3. Let $A=\left(A_{1}, \ldots, A_{n}\right)$ and $B=\left(B_{1}, \ldots, B_{k}\right)$ be commuting tuples on a vector space $V$ such that the union $A \oplus B$ commutes as well. Suppose that the induced homomorphism

$$
H_{*}\left(B_{j}\right): H_{*}\left(A \oplus\left(B_{1}, \ldots, B_{j-1}\right), V\right) \rightarrow H_{*}\left(A \oplus\left(B_{1}, \ldots, B_{j-1}\right), V\right)
$$

vanishes for each $j \in\{1, \ldots, k\}$. We then have the identity

$$
\operatorname{Dim}_{\mathbb{C}} H_{q}(A \oplus B, V)=\sum_{p=0}^{k}\left(\begin{array}{l}
k \\
p
\end{array}\right) \operatorname{Dim}_{\mathbb{C}} H_{q-p}(A, V)
$$

of dimensions over $\mathbb{C}$ for all $q \in\{0, \ldots, n+k\}$.

Proof. The proof runs by induction on the number of elements in $B$. The statement is obviously true when there are no elements in $B$. Thus, suppose that it is valid for some $k_{0} \in \mathbb{N} \cup\{0\}$. By Lemma 2.4 we get the existence of a long exact sequence

$$
\begin{aligned}
& \ldots \longleftarrow H_{q-1}\left(A \oplus p_{k_{0}}(B)\right) \longleftarrow H_{q}\left(A \oplus p_{k_{0}+1}(B)\right) \longleftarrow H_{q}\left(A \oplus p_{k_{0}}(B)\right) \\
& \uparrow H_{q}\left(B_{k_{0}+1}\right) \\
& \ldots \quad \longrightarrow H_{q+1}\left(A \oplus p_{k_{0}+1}(B)\right) \longrightarrow H_{q}\left(A \oplus p_{k_{0}}(B)\right)
\end{aligned}
$$

Now, since $H_{*}\left(B_{k_{0}+1}\right): H_{*}\left(A \oplus\left(B_{1}, \ldots, B_{k_{0}}\right)\right) \rightarrow H_{*}\left(A \oplus\left(B_{1}, \ldots, B_{k_{0}}\right)\right)$ is trivial we can conclude that

$\operatorname{Dim}_{\mathbb{C}} H_{q}\left(A \oplus p_{k_{0}+1}(B), V\right)=\operatorname{Dim}_{\mathbb{C}} H_{q}\left(A \oplus p_{k_{0}}(B), V\right)+\operatorname{Dim}_{\mathbb{C}} H_{q-1}\left(A \oplus p_{k_{0}}(B), V\right)$

for all $q \in\left\{0, \ldots, n+k_{0}+1\right\}$. The induction hypothesis then implies that

$$
\begin{aligned}
\operatorname{Dim}_{\mathbb{C}} H_{q}\left(A \oplus p_{k_{0}+1}(B), V\right) & =\sum_{p=0}^{k_{0}+1}\left(\left(\begin{array}{c}
k_{0} \\
p
\end{array}\right)+\left(\begin{array}{c}
k_{0} \\
p-1
\end{array}\right)\right) \operatorname{Dim}_{\mathbb{C}} H_{q-p}(A, V) \\
& =\sum_{p=0}^{k_{0}+1}\left(\begin{array}{c}
k_{0}+1 \\
p
\end{array}\right) \operatorname{Dim} H_{q-p}(A, V),
\end{aligned}
$$

which is the desired identity when $B$ has $k_{0}+1$ elements. This proves the lemma.

The above results can be combined into:

Lemma 5.4. For each $q \in\{0, \ldots, n+m\}$ the following equalities hold:

$$
\begin{aligned}
& \operatorname{Dim}_{\mathbb{C}} H_{q}\left((z-\lambda) \oplus g, \mathscr{H}_{\lambda}\right)=\sum_{p=0}^{n}\left(\begin{array}{l}
n \\
p
\end{array}\right) \operatorname{Dim}_{\mathbb{C}} H_{q-p}\left(g, \mathscr{H}_{\lambda}\right) \\
& \operatorname{Dim}_{\mathbb{C}} H_{q}\left((z-\lambda) \oplus g, \mathscr{H}_{\lambda}\right)=\sum_{p=0}^{m}\left(\begin{array}{c}
m \\
p
\end{array}\right) \operatorname{Dim}_{\mathbb{C}} H_{q-p}(A-\lambda, \mathscr{H}) .
\end{aligned}
$$


Proof. Remark the existence of an isomorphism $H_{*}\left(z-\lambda, \mathscr{H}_{\lambda}\right) \cong H_{*}(A-\lambda, \mathscr{H})$. Indeed, we have that $H_{*}(A-\lambda, \mathscr{H})_{\lambda} \cong H_{*}(A-\lambda, \mathscr{H})$ since each holomorphic function $f \in \mathcal{O}(\operatorname{Sp}(A))$ acts by scalar multiplication with $f(\lambda) \in \mathbb{C}$ on the homology group $H_{*}(A-\lambda, \mathscr{H})$.

The desired identities now follow immediately from the above lemmas. Notice that $H_{*}\left((z-\lambda) \oplus g, \mathscr{H}_{\lambda}\right) \cong H_{*}\left(g \oplus(z-\lambda), \mathscr{H}_{\lambda}\right)$ by the symmetry of the Koszul homology groups. See f. ex. [8, Theorem 3.2.3].

Let $R(n): \mathbb{N}_{0}^{m+1} \rightarrow \mathbb{N}_{0}^{n+m+1}$ and $R(m): \mathbb{N}_{0}^{n+1} \rightarrow \mathbb{N}_{0}^{n+m+1}$ denote the linear maps represented by the matrices

$$
\begin{aligned}
R_{i j}(n):=\left(\begin{array}{c}
n \\
i-j
\end{array}\right) & j \in\{0, \ldots, m\}, i \in\{0, \ldots, n+m\} \text { and } \\
R_{i j}(m):=\left(\begin{array}{c}
m \\
i-j
\end{array}\right) & j \in\{0, \ldots, n\}, i \in\{0, \ldots, n+m\} .
\end{aligned}
$$

Remark that for $k \in \mathbb{N}_{0}$ and $p \in \mathbb{Z}$ we use the convention $\left(\begin{array}{l}k \\ p\end{array}\right):=0$ whenever $p<0$ or $p>k$. Remark also that $m \geq n$ by our general assumption. Define furthermore the linear map $L(n): \mathbb{N}_{0}^{n+m+1} \rightarrow \mathbb{N}_{0}^{m+1}$ by the matrix representation

$$
L_{i j}(n):=\left\{\begin{array}{cc}
(-1)^{i-j}\left(\begin{array}{cc}
n+i-j-1 \\
i-j
\end{array}\right) & \text { for } \quad i \geq j \\
0 & \text { for } i<j,
\end{array}\right.
$$

where $j \in\{0, \ldots, n+m\}$ and $i \in\{0, \ldots, m\}$.

Lemma 5.5. The composition $L(n) \circ R(m): \mathbb{N}_{0}^{n+1} \rightarrow \mathbb{N}_{0}^{m+1}$ is represented by the matrix with entries

$$
(L(n) \circ R(m))_{i j}=\left(\begin{array}{c}
m-n \\
i-j
\end{array}\right) \quad j \in\{0, \ldots, n\}, i \in\{0, \ldots, m\} .
$$

In particular we have that $L(n) \circ R(n)=\mathrm{Id}: \mathbb{N}_{0}^{n+1} \rightarrow \mathbb{N}_{0}^{n+1}$.

Proof. Let $j \in\{0, \ldots, n\}$ and $i \in\{0, \ldots, m\}$. Compute as follows,

$$
\begin{aligned}
(L(n) \circ R(m))_{i j} & =\sum_{k=0}^{i}(-1)^{i-k}\left(\begin{array}{c}
n+i-k-1 \\
i-k
\end{array}\right) \cdot\left(\begin{array}{c}
m \\
k-j
\end{array}\right) \\
& =\left\{\begin{array}{cl}
\sum_{k=j}^{i}(-1)^{i-k}\left(\begin{array}{c}
n+i-k-1 \\
i-k
\end{array}\right) \cdot\left(\begin{array}{c}
m \\
k-j
\end{array}\right) & \text { for } i \geq j \\
0 & \text { for } i<j
\end{array}\right. \\
& =\left\{\begin{array}{cc}
\sum_{p=0}^{i-j}(-1)^{p}\left(\begin{array}{c}
n+p-1 \\
p
\end{array}\right) \cdot\left(\begin{array}{c}
m \\
i-j-p
\end{array}\right) & \text { for } i \geq j \\
0 & \text { for } i<j .
\end{array}\right.
\end{aligned}
$$


The desired result is then a consequence of the combinatorial identity

$$
\sum_{p=0}^{i-j}(-1)^{p}\left(\begin{array}{c}
n+p-1 \\
p
\end{array}\right)\left(\begin{array}{c}
m \\
i-j-p
\end{array}\right)=\left(\begin{array}{c}
m-n \\
i-j
\end{array}\right) \quad i \geq j .
$$

We are now ready to prove the main result of this section.

Proposition 5.6. Suppose that $g(A)$ is Fredholm and that $\lambda \in Z(g)$ is a regular point for $\left(g_{1}, \ldots, g_{n}\right): \operatorname{Sp}(A) \rightarrow \mathbb{C}^{n}$. We then have the equality

$$
\operatorname{Dim}_{\mathbb{C}} H_{q}\left(g, \mathscr{H}_{\lambda}\right)=\sum_{p=0}^{m-n}\left(\begin{array}{c}
m-n \\
p
\end{array}\right) \operatorname{Dim}_{\mathbb{C}} H_{q-p}(A-\lambda, \mathscr{H})
$$

for each $q \in\{0, \ldots, m\}$.

Proof. Let $\eta \in \mathbb{N}_{0}^{n+m+1}, \xi \in \mathbb{N}_{0}^{m+1}$ and $\zeta \in \mathbb{N}_{0}^{n+1}$ be defined by

$$
\begin{aligned}
\eta_{p} & :=\operatorname{Dim}_{\mathbb{C}} H_{p}\left((z-\lambda) \oplus g, \mathscr{H}_{\lambda}\right) & & p=0, \ldots, n+m \\
\xi_{q} & :=\operatorname{Dim}_{\mathbb{C}} H_{q}\left(g, \mathscr{H}_{\lambda}\right) & q & =0, \ldots, m \\
\zeta_{k} & :=\operatorname{Dim}_{\mathbb{C}} H_{k}(A-\lambda, \mathscr{H}) & k & =0, \ldots, n .
\end{aligned} \text { and }
$$

It then follows from Lemma 5.4 that

$$
R(n)(\xi)=\eta=R(m)(\zeta) .
$$

In particular we get that $L(n) R(n)(\xi)=L(n) R(m)(\zeta)$ and the proposition is proved by an application of Lemma 5.5.

As an application of Proposition 5.6 we obtain the local index theorem in the regular case.

Theorem 5.7. Suppose that $g(A)$ is Fredholm and that $\lambda \in Z(g)$ is a regular point for $\left(g_{1}, \ldots, g_{n}\right): \operatorname{Sp}(A) \rightarrow \mathbb{C}^{n}$. Then

$$
\operatorname{Ind}_{\lambda}(g(A))=\left\{\begin{array}{ccc}
\operatorname{Ind}(A-\lambda) & \text { for } & m=n \\
0 & \text { for } & m>n
\end{array}\right.
$$

Proof. The result follows immediately from Proposition 5.6 when $m=n$ since $\operatorname{Dim}_{\mathbb{C}} H_{q}\left(g, \mathscr{H}_{\lambda}\right)=\operatorname{Dim}_{\mathbb{C}} H_{q}(A-\lambda, \mathscr{H})$ for all $q \in\{0, \ldots, n\}$ in this case. Notice that $H_{q}\left(g, \mathscr{H}_{\lambda}\right) \cong H_{q}(g(A), \mathscr{H})(\lambda)$ by Proposition 4.8 . 
Thus suppose that $m>n$. By Proposition 5.6 we have that

$$
\begin{aligned}
\operatorname{Ind}_{\lambda}(g(A)) & =\sum_{q=0}^{m}(-1)^{q+1} \operatorname{Dim}_{\mathbb{C}} H_{q}\left(g, \mathscr{H}_{\lambda}\right) \\
& =\sum_{q=0}^{m}(-1)^{q+1} \sum_{p=0}^{m-n}\left(\begin{array}{c}
m-n \\
p
\end{array}\right) \operatorname{Dim}_{\mathbb{C}} H_{q-p}(A-\lambda, \mathscr{H}) \\
& =\sum_{p=0}^{m-n}(-1)^{p}\left(\begin{array}{c}
m-n \\
p
\end{array}\right) \sum_{j=0}^{m-p}(-1)^{j+1} \operatorname{Dim}_{\mathbb{C}} H_{j}(A-\lambda, \mathscr{H}) \\
& =\left(\sum_{p=0}^{m-n}(-1)^{p}\left(\begin{array}{c}
m-n \\
p
\end{array}\right)\right) \cdot\left(\sum_{j=0}^{n}(-1)^{j+1} \operatorname{Dim}_{\mathbb{C}} H_{j}(A-\lambda, \mathscr{H})\right) \\
& =0 .
\end{aligned}
$$

This ends the proof of the theorem.

\section{The global index theorem}

Throughout this section the following condition will be in effect:

Let $g: \operatorname{Sp}(A) \rightarrow \mathbb{C}^{m}$ be a holomorphic map on the Taylor spectrum of the commuting tuple $A=\left(A_{1}, \ldots, A_{n}\right)$ of bounded operators such that $g(A)$ is Fredholm.

We will be proving a global index theorem which expresses the index of $g(A)$ in terms of the locally constant index function associated with the "variables" $A$ and local degrees of the "symbol" $g \in \mathcal{O}(\operatorname{Sp}(A))$ near the set of common zeroes. The proof will rely on our computation of the local indices of $g(A)$ for regular zeroes (Theorem 5.7) together with the homotopy invariance of the Fredholm index.

As in the last section we let $p_{n}(g):=\left(g_{1}, \ldots, g_{n}\right)$ whenever $m \geq n$.

Notation 6.1. The notation $C_{p_{n}(g)} \subseteq \operatorname{Sp}(A)$ refers to the set of critical points for $p_{n}(g): \operatorname{Sp}(A) \rightarrow \mathbb{C}^{n}$, thus the points for which the determinant of the Jacobian matrix $\operatorname{det}\left(\frac{\partial g_{i}}{\partial z_{j}}\right): \operatorname{Sp}(A) \rightarrow \mathbb{C}$ vanishes.

It follows by Sard's Lemma that the image $p_{n}(g)\left(C_{p_{n}(g)}\right) \subseteq \mathbb{C}^{n}$ has Lebesgue measure zero. See [15, Theorem 3.1].

Theorem 6.2. Suppose that $g(A)$ is Fredholm and that $m>n$. Then $\operatorname{Ind}(g(A))=0$.

Proof. By the homotopy invariance of the index, [2, Theorem 3], we can find an open neighborhood $U$ of $0 \in \mathbb{C}^{m}$ such that $g(A)-z=(g-z)(A)$ is Fredholm with $\operatorname{Ind}(g(A)-z)=\operatorname{Ind}(g(A))$ for all $z \in U$. Since $p_{n}(g)\left(C_{p_{n}(g)}\right)$ has measure 
zero, we may thus assume, without loss of generality, that $0 \notin p_{n}(g)\left(C_{p_{n}(g)}\right)$. This clearly implies that every $\lambda \in Z(g)$ is a regular point for $p_{n}(g): \operatorname{Sp}(A) \rightarrow \mathbb{C}^{n}$. By Theorem 5.7 we then get that $\operatorname{Ind}_{\lambda}(g(A))=0$ for all $\lambda \in Z(g)$. The result of the theorem now follows by an application of Proposition 3.6.

Notation 6.3. Let $\Omega:=\operatorname{Int}(\operatorname{Sp}(A))$ denote the interior of the Taylor spectrum.

Suppose now that $m=n$ and that $\lambda \in Z(g) \cap \Omega$. By Theorem 3.3 we have that $Z(g)$ is finite and we can thus find an open ball $\mathbb{B}_{\varepsilon} \subseteq \Omega$ such that $Z(g) \cap \mathbb{B}_{\varepsilon}=\{\lambda\}$.

Notation 6.4. The notation $\operatorname{deg}_{\lambda}(g):=\operatorname{deg}\left(0, g, \mathbb{B}_{\varepsilon}\right) \in \mathbb{N}$ refers to the degree of the holomorphic map $g: \mathbb{B}_{\varepsilon} \rightarrow \mathbb{R}^{2 n}$ at the value 0 (where $\mathbb{C}^{n}$ is identified with $\mathbb{R}^{2 n}$ ). This integer will be called the local degree of $g$ at $\lambda$.

For later use we record the following result which can be deduced from [3, Proposition 2.4].

Lemma 6.5. The local degree $\operatorname{deg}_{\lambda}(g)$ coincides with the vector space dimension of the quotient $\mathcal{O}_{\lambda} / g \mathcal{O}_{\lambda}:=\mathcal{O}_{\lambda} /\left(g_{1} \mathcal{O}_{\lambda}+\ldots+g_{n} \mathcal{O}_{\lambda}\right)$ for each $\lambda \in Z(g) \cap \Omega$.

The global index theorem can now be proved in the main case of interest. See also [4, Theorem 10.3.13].

Theorem 6.6. Suppose that $g(A)$ is Fredholm and that $m=n$. Then

$$
\operatorname{Ind}(g(A))=\sum_{\lambda \in Z(g) \cap \Omega} \operatorname{deg}_{\lambda}(g) \cdot \operatorname{Ind}(A-\lambda) .
$$

Proof. Let us choose a small open ball $\mathbb{B}_{\varepsilon(\lambda)} \subseteq \mathbb{C}^{n}$ for each $\lambda \in Z(g)$ such that the following conditions hold:

(1) $\lambda \in \mathbb{B}_{\varepsilon(\lambda)}$.

(2) $\mathbb{B}_{\varepsilon(\lambda)} \cap \mathbb{B}_{\varepsilon(\mu)}=\emptyset$ whenever $\lambda \neq \mu$.

(3) $\mathbb{B}_{\varepsilon(\lambda)} \cap \operatorname{Sp}_{\text {ess }}(A)=\emptyset$ for all $\lambda \in Z(g)$.

(4) $\mathbb{B}_{\varepsilon(\lambda)} \subseteq \Omega$ for all $\lambda \in Z(g) \cap \Omega$.

Recall that the image $g\left(C_{g}\right)$ of the set of critical points for $g$ has measure zero. We can thus find a sequence $\left\{\alpha_{k}\right\}$ of elements in $\mathbb{C}^{n}$ which converges to zero such that the set of common zeroes $Z\left(g-\alpha_{k}\right)$ consists entirely of regular points for all $k \in \mathbb{N}$.

Let us fix some element $\alpha:=\alpha_{k_{0}}$ from the above sequence.

By the homotopy invariance of the index and of the degree we may assume that

$$
\operatorname{Ind}((g-\alpha)(A))=\operatorname{Ind}(g(A)) \text { and } \operatorname{deg}\left(0, g-\alpha, \mathbb{B}_{\varepsilon(\lambda)}\right)=\operatorname{deg}_{\lambda}(g)
$$

for all $\lambda \in Z(g) \cap \Omega$. See [2, Theorem 3] and [15, Theorem 3.16].

Furthermore, by a compactness argument we may assume that

$$
Z(g-\alpha)=Z(g-\alpha) \cap \mathbb{B} \text { where } \mathbb{B}:=\cup_{\lambda \in Z(g)} \mathbb{B}_{\varepsilon(\lambda)} .
$$


The result of Theorem 5.7 then implies that

$$
\begin{aligned}
\operatorname{Ind}(g(A))=\operatorname{Ind}((g-\alpha)(A)) & =\sum_{\mu \in Z(g-\alpha)} \operatorname{Ind}(A-\mu) \\
& =\sum_{\lambda \in Z(g)} \sum_{\mu \in Z(g-\alpha) \cap \mathbb{B}_{\varepsilon(\lambda)}} \operatorname{Ind}(A-\mu) .
\end{aligned}
$$

Suppose that $\lambda \in Z(g) \cap \Omega$. Since $Z(g-\alpha) \cap C_{g}=\emptyset$, the degree $\operatorname{deg}(0, g-$ $\left.\alpha, \mathbb{B}_{\varepsilon(\lambda)}\right) \in \mathbb{N}$ is nothing but the number of zeroes of the function $g-\alpha: \mathbb{B}_{\varepsilon(\lambda)} \rightarrow \mathbb{C}^{n}$, thus

$$
\operatorname{deg}_{\lambda}(g)=\operatorname{deg}\left(0, g-\alpha, \mathbb{B}_{\varepsilon(\lambda)}\right)=\left|Z(g-\alpha) \cap \mathbb{B}_{\varepsilon(\lambda)}\right| .
$$

Furthermore, since $\mathbb{B}_{\varepsilon(\lambda)} \cap \operatorname{Sp}_{\text {ess }}(A)=\emptyset$ we have that $\operatorname{Ind}(A-\mu)=\operatorname{Ind}(A-\lambda)$ for all $\mu \in \mathbb{B}_{\varepsilon(\lambda)}$. This allows us to compute as follows:

$$
\sum_{\mu \in Z(g-\alpha) \cap \mathbb{B}_{\varepsilon(\lambda)}} \operatorname{Ind}(A-\mu)=\left|Z(g-\alpha) \cap \mathbb{B}_{\varepsilon(\lambda)}\right| \cdot \operatorname{Ind}(A-\lambda)=\operatorname{deg}_{\lambda}(g) \cdot \operatorname{Ind}(A-\lambda) .
$$

To finish the proof of the global index theorem we therefore only need to verify the identity

$$
\sum_{\mu \in Z(g-\alpha) \cap \mathbb{B}_{\varepsilon}(\lambda)} \operatorname{Ind}(A-\mu)=0
$$

whenever $\lambda \in Z(g) \cap \partial \operatorname{Sp}(A)$. But for such a $\lambda, \operatorname{Ind}(A-\lambda)=0$, and the desired identity follows since $\operatorname{Ind}(A-\lambda)=\operatorname{Ind}(A-\mu)$ for all $\mu \in \mathbb{B}_{\varepsilon}(\lambda)$.

The next result will only rely on the finiteness of the zero set $Z(g)$ and some considerations on dimensions.

Theorem 6.7. Suppose that $g(A)$ is Fredholm and that $m<n$. Then $Z(g) \subseteq$ $\operatorname{Sp}(A) \backslash \operatorname{cl}(\Omega)$.

Proof. Suppose for contradiction that $Z(g) \cap \operatorname{cl}(\Omega) \neq \emptyset$ and let $\lambda \in Z(g) \cap \operatorname{cl}(\Omega)$.

Suppose first that $\lambda \in \Omega$. From Theorem 3.3 we know that $Z(g)$ is a finite set. In particular we get that $\lambda$ is an isolated point. But this is a contradiction since the analytic dimension of $\mathbb{C}^{n}$ at $\lambda$ is $n$ whereas the number of coordinates for $g=\left(g_{1}, \ldots, g_{m}\right)$ is strictly less than $n$. See [7, Chapter 5, §3.1].

Suppose next that $\lambda \in \partial \Omega$. We can then find a sequence $\left\{\lambda_{k}\right\}$ of elements in $\Omega$ which converges to $\lambda$. Since $g(\lambda)=0$ and $g: \operatorname{Sp}(A) \rightarrow \mathbb{C}^{m}$ is continuous we get that $\left\{g\left(\lambda_{k}\right)\right\}$ converges to zero. Furthermore, since $g(A)$ is Fredholm we have that $0 \notin g\left(\operatorname{Sp}_{\text {ess }}(A)\right)=\operatorname{Sp}_{\text {ess }}(g(A))$. By the compactness of $\operatorname{Sp}_{\text {ess }}(A) \subseteq \mathbb{C}^{n}$ we can thus find an open neighborhood $U$ of zero such that $U \cap \operatorname{Sp}_{\text {ess }}(g(A))=\emptyset$.

Choose a $k \in \mathbb{N}$ such that $\mu:=g\left(\lambda_{k}\right) \in U$. The function $h:=g-\mu$ : $\operatorname{Sp}(A) \rightarrow \mathbb{C}^{m}$ is now holomorphic and the set $Z(h) \cap \Omega$ is non-trivial. Furthermore, since $0 \notin h\left(\operatorname{Sp}_{\text {ess }}(A)\right)=\operatorname{Sp}_{\text {ess }}(g(A))-\mu$ we conclude that $h(A)$ is Fredholm. The above argument then leads to a contradiction in this case as well. 
Remark 6.8. It is in general not true that $\operatorname{Ind}(g(A))=0$ when $m<n$. As an example, suppose that $n=2$ and that $A=(B, B)$ where $B: \mathscr{H} \rightarrow \mathscr{H}$ is a Fredholm operator with $\operatorname{Ind}(B) \neq 0$. If $g: \mathbb{C}^{2} \rightarrow \mathbb{C}$ is the projection onto the first coordinate we get that $\operatorname{Ind}(g(A))=\operatorname{Ind}(B)$.

\section{Analytic localization and reciprocity of local indices}

In this section the following notational convention will be used:

Let $B=\left(B_{1}, \ldots, B_{n}\right)$ be an extra commuting tuple of the same length as $A=\left(A_{1}, \ldots, A_{n}\right)$ but acting on a possibly different Hilbert space $\mathscr{G}$. The letter $\mathscr{K}:=\mathscr{H} \widehat{\otimes} \mathscr{G}$ will refer to the Hilbert space completion of the tensor product of our two Hilbert spaces.

The purpose of this section is twofold. We will investigate an analytic version of the algebraic localization procedure introduced in Section 4. Furthermore, we will prove a reciprocity formula which relates the local indices of the two independent commuting tuples $A$ and $B$.

Before presenting these results, we need some preliminary lemmas. They are mainly concerned with the behaviour of indices and spectra under the tensor product operation.

Let $A \otimes 1:=\left(A_{1} \otimes 1, \ldots, A_{n} \otimes 1\right)$ and $1 \otimes B:=\left(1 \otimes B_{1}, \ldots, 1 \otimes B_{n}\right)$ act on the Hilbert space $\mathscr{K}:=\mathscr{H} \widehat{\otimes} \mathscr{G}$. Remark that the union $(A \otimes 1) \oplus(1 \otimes B)$ is a commuting tuple.

Lemma 7.1. There is an inclusion of spectra,

$$
\mathrm{Sp}((A \otimes 1) \oplus(1 \otimes B)) \subseteq \operatorname{Sp}(A) \times \operatorname{Sp}(B) .
$$

Furthermore, for each $f \in \mathcal{O}(\operatorname{Sp}(A))$ and $h \in \mathcal{O}(\operatorname{Sp}(B))$,

$$
f(A \otimes 1)=f(A) \otimes 1 \text { and } h(1 \otimes B)=1 \otimes h(B) .
$$

Proof. Let $(\lambda, \mu) \notin \operatorname{Sp}(A) \times \operatorname{Sp}(B)$ and let us show that $(\lambda, \mu) \notin \operatorname{Sp}((A \otimes 1) \oplus$ $(1 \otimes B))$. Without loss of generality we may assume that $\lambda \notin \mathrm{Sp}(A)$. It is then sufficient to prove that the Koszul complex $K(A \otimes 1-\lambda, \mathscr{H} \widehat{\otimes} \mathscr{G})$ is exact. But this is true since the Koszul complex $K(A-\lambda, \mathscr{H})$ is exact and since the functor $\cdot \widehat{\otimes} \mathscr{G}$ preserves short exact sequences of Hilbert spaces.

Let us show that $f(A \otimes 1)=f(A) \otimes 1$. The other identity in (7.1) follows by a similar argument. Let $\gamma \in \mathscr{L}(\mathscr{G}, \mathbb{C})$ be a linear functional on $\mathscr{G}$. It is then enough to prove the identity

$$
f(A)(1 \otimes \gamma)=(1 \otimes \gamma) f(A \otimes 1) .
$$


But this follows from Proposition 2.12 since $A_{i}(1 \otimes \gamma)=(1 \otimes \gamma)\left(A_{i} \otimes 1\right)$ for all $i \in\{1, \ldots, n\}$. Notice that $\operatorname{Sp}(A \otimes 1) \subseteq \operatorname{Sp}(A)$ by an application of the first part of the lemma.

The notation $A \otimes 1-1 \otimes B$ refers to the commuting tuple $A \otimes 1-1 \otimes B:=$ $\left(A_{1} \otimes 1-1 \otimes B_{1}, \ldots, A_{n} \otimes 1-1 \otimes B_{n}\right)$.

Lemma 7.2. Let $C$ be an extra commuting tuple on $\mathscr{K}$ such that $(A \otimes 1-1 \otimes B) \oplus C$ commutes. Let $f: \operatorname{Sp}(A) \cup \operatorname{Sp}(B) \rightarrow \mathbb{C}^{m}$ be holomorphic. Then there exists an isomorphism of Koszul homology groups

$H_{*}((A \otimes 1-1 \otimes B) \oplus C \oplus(f(A) \otimes 1), \mathscr{K}) \cong H_{*}((A \otimes 1-1 \otimes B) \oplus C \oplus(1 \otimes f(B)), \mathscr{K})$.

Proof. We use the short notation $f(A):=f(A) \otimes 1$ and $f(B):=1 \otimes f(B)$ as well as $D:=(A \otimes 1-1 \otimes B) \oplus C$.

Without loss of generality we may assume that $m=1$. By Lemma 2.4 we have two long exact sequences of homology groups

$$
\begin{aligned}
& \ldots \longrightarrow H_{*+1}(D \oplus f(A), \mathscr{K}) \longrightarrow H_{*}(D, \mathscr{K}) \stackrel{H_{*}(f(A))}{\longrightarrow} \quad H_{*}(D, \mathscr{K}) \\
& \downarrow
\end{aligned}
$$

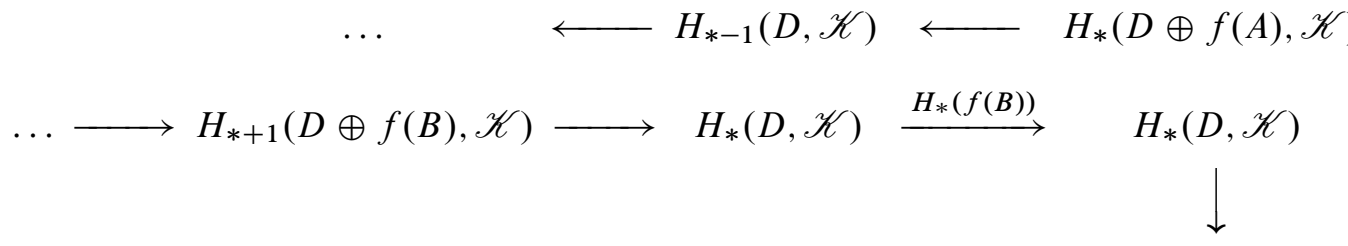

$$
\begin{aligned}
& \ldots \quad \longleftarrow H_{*-1}(D, \mathscr{K}) \longleftarrow H_{*}(D \oplus f(B), \mathscr{K})
\end{aligned}
$$

However, by an application of [4, Proposition 2.5.9] and Lemma 7.1 we get that the linear maps $H_{*}(f(A) \otimes 1)$ and $H_{*}(1 \otimes f(B))$ are identical. This implies the result of the lemma since we are working in the category of vector spaces over $\mathbb{C}$. Notice however that the isomorphism which we obtain is not canonical since it depends on the choice of complementary subspaces.

Lemma 7.3. Suppose that $A$ and $B$ are Fredholm. Then the commuting tuple $(A \otimes 1) \oplus(1 \otimes B)$ is Fredholm on $\mathscr{H} \widehat{\otimes} \mathscr{G}$ with index

$$
\operatorname{Ind}((A \otimes 1) \oplus(1 \otimes B))=-\operatorname{Ind}(A) \cdot \operatorname{Ind}(B) .
$$

Proof. By Proposition 2.20 it suffices to show that $H_{p}\left(A \otimes 1, H_{q}((1 \otimes B), \mathscr{K})\right)$ is finite dimensional for each $p, q \in\{0, \ldots, n\}$ and that

$$
\sum_{p, q}(-1)^{p+q+1} \operatorname{Dim}\left(H_{p}\left(A \otimes 1, H_{q}((1 \otimes B), \mathscr{K})\right)\right)=\operatorname{Ind}(A) \cdot \operatorname{Ind}(B) .
$$


Let $p, q \in\{0, \ldots, n\}$. Using the Fredholmness of $A$ and $B$ together with the exactness of the Hilbert space tensor product we get the isomorphisms

$$
\begin{aligned}
H_{p}\left(A \otimes 1, H_{q}((1 \otimes B), \mathscr{K})\right) & \cong H_{p}\left(A \otimes 1, \mathscr{H} \otimes H_{q}(B, \mathscr{G})\right) \\
& \cong H_{p}(A, \mathscr{H}) \otimes H_{q}(B, \mathscr{G}) .
\end{aligned}
$$

See also the proof of Proposition 7.5. This shows that $H_{*}\left(A \otimes 1, H_{*}((1 \otimes B), \mathscr{K})\right)$ is finite dimensional and thus that $(A \otimes 1) \oplus(1 \oplus B)$ is Fredholm.

Furthermore, the identity in (7.2) implies that

$$
\begin{aligned}
\sum_{p, q}(-1)^{p+q+1} \operatorname{Dim} & \left(H_{p}\left(A \otimes 1, H_{q}((1 \otimes B), \mathscr{K})\right)\right) \\
& =\sum_{p}(-1)^{p} \operatorname{Dim}\left(H_{p}(A, \mathscr{H})\right) \cdot \sum_{q}(-1)^{q+1} \operatorname{Dim}\left(H_{q}(B, \mathscr{G})\right) \\
& =-\operatorname{Ind}(A) \cdot \operatorname{Ind}(B),
\end{aligned}
$$

and the lemma is proved.

Lemma 7.4. Let $V$ be a vector space of finite dimension over $\mathbb{C}$. Suppose that $A=\left(A_{1}, \ldots, A_{n}\right)$ is Fredholm on $\mathscr{H}$ and let $C=\left(C_{1}, \ldots, C_{n}\right)$ be a commuting tuple on $V$ with $C_{i}$ nilpotent for all $i \in\{1, \ldots, n\}$. We then have the identity

$$
\operatorname{Ind}(A \otimes 1+1 \otimes C)=\operatorname{Ind}(A) \cdot \operatorname{Dim}(V),
$$

where the commuting tuple $A \otimes 1+1 \otimes C$ acts on the Hilbert space $\mathscr{H} \otimes V$.

Proof. We argue by induction on the dimension of $V$.

Suppose that $\operatorname{Dim}(V)=1$. This implies that $C$ is trivial. Let $\xi \in V$ be a non-trivial vector. The isomorphism of Hilbert space $\mathscr{H} \otimes V \cong \mathscr{H}$ given by $\eta \otimes \alpha \cdot \xi \mapsto \alpha \cdot \eta, \eta \in \mathscr{H}, \alpha \in \mathbb{C}$, then induces an isomorphism of Koszul homology groups $H_{*}(A \otimes 1, \mathscr{H} \otimes V) \cong H_{*}(A, \mathscr{H})$. This proves the statement in this case.

Suppose that the statement is true for $\operatorname{Dim}(V)=k$ for some $k \geq 1$ and suppose that $\operatorname{Dim}(V)=k+1$. Since $C$ is nilpotent on $V$ there exists a non-trivial vector $\xi \in V$ with $C_{i}(\xi)=0$ for all $i \in\{1, \ldots, n\}$. See Theorem 2.5. Let $W:=\mathbb{C} \cdot \xi \subseteq V$ denote the span of $\xi$ in $V$. There is a long exact sequence of homology groups

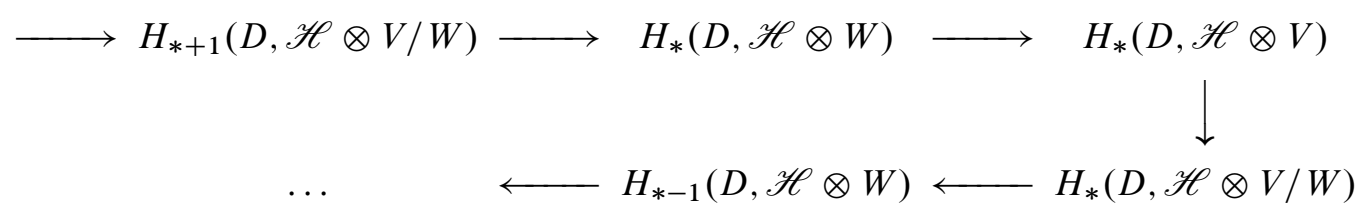

where $D:=A \otimes 1+1 \otimes C$. By the induction hypothesis and the induction start we get that $\operatorname{Ind}\left(\left.D\right|_{\mathscr{H}} \otimes W\right)=\operatorname{Ind}(A)$ and $\operatorname{Ind}\left(\left.D\right|_{\mathscr{H} \otimes V / W}\right)=\operatorname{Dim}(V / W) \cdot \operatorname{Ind}(A)$. This proves the induction step by the additivity of the Euler characteristic. 
The following result shows that the passage from $\mathscr{H}$ to the tensor product $\mathscr{H} \widehat{\otimes} \mathscr{G}$ can be used as an analytic counterpart of the algebraic localization at the zeroes of a holomorphic function as far as index computations are concerned. See also Lemma 8.1. This will turn out to be important for the proof of our local index theorem.

Proposition 7.5. Let $g: \operatorname{Sp}(A) \rightarrow \mathbb{C}^{m}$ be holomorphic. Suppose that $g(A)$ is Fredholm and that $\lambda-B$ is Fredholm for all $\lambda \in Z(g)$. Then the commuting tuple $(A \otimes 1-1 \otimes B) \oplus(g(A) \otimes 1)$ is Fredholm on $\mathscr{H} \widehat{\otimes} \mathscr{G}$ and the index is given by

$$
\operatorname{Ind}((A \otimes 1-1 \otimes B) \oplus(g(A) \otimes 1))=-\sum_{\lambda \in Z(g)} \operatorname{Ind}_{\lambda}(g(A)) \cdot \operatorname{Ind}(\lambda-B) .
$$

Proof. By Proposition 2.20 it is enough to show that $H_{p}(A \otimes 1-1 \otimes B$, $H_{q}(g(A) \otimes 1, \mathscr{K})$ is finite dimensional for all $p \in\{0, \ldots, n\}$ and $q \in\{0, \ldots, m\}$ and that

$$
\begin{aligned}
\sum_{p, q}(-1)^{p+q+1} \operatorname{Dim}_{\mathbb{C}} H_{p}(A \otimes 1-1 & \left.\otimes B, H_{q}(g(A) \otimes 1, \mathscr{K})\right) \\
& =-\sum_{\lambda \in Z(g)} \operatorname{Ind}_{\lambda}(g(A)) \cdot \operatorname{Ind}(\lambda-B) .
\end{aligned}
$$

Let us fix a $q \in\{0, \ldots, m\}$. Note that $H_{q}(g(A) \otimes 1, \mathscr{K}) \cong H_{q}(g(A), \mathscr{H}) \otimes \mathscr{G}$. To see this, it suffices to recall that the functor $\cdot \widehat{\otimes} \mathscr{G}$ sends short exact sequences of Hilbert spaces to short exact sequences of Hilbert spaces. Furthermore, the image of the differential of the Koszul complex $K_{*}(g(A), \mathscr{H})$ is closed by the Fredholmness assumption on $g(A)$.

Using the Fredholmness of $g(A)$ one more time, we can decompose $H_{q}(g(A), \mathscr{H})$ into the generalized eigenspaces

$$
H_{q}(g(A), \mathscr{H}) \cong \bigoplus_{\lambda \in Z(g)} H_{q}(g(A), \mathscr{H})(\lambda)
$$

for the commuting tuple $H_{q}(A)$.

Let $\lambda \in Z(g)$. Since $\lambda-B$ is Fredholm on $\mathscr{G}$ and $H_{q}(A)(\lambda)-\lambda$ is nilpotent on $H_{q}(g(A), \mathscr{H})(\lambda)$ we get from Lemma 7.4 that

$$
\begin{aligned}
\operatorname{Ind}\left(H_{q}(A)(\lambda) \otimes 1-1 \otimes B\right) & =\operatorname{Ind}\left(\left(H_{q}(A)(\lambda)-\lambda\right) \otimes 1+1 \otimes(\lambda-B)\right) \\
& =\operatorname{Dim}_{\mathbb{C}}\left(H_{q}(g(A), \mathscr{H})(\lambda)\right) \cdot \operatorname{Ind}(\lambda-B),
\end{aligned}
$$

where $H_{q}(A)(\lambda) \otimes 1-1 \otimes B$ acts on the Hilbert space $H_{q}(g(A), \mathscr{H})(\lambda) \otimes \mathscr{G}$. 
Combining the above identities we get that

$$
\begin{aligned}
\sum_{q}(-1)^{q} \cdot \operatorname{Ind} & \left(H_{q}(A) \otimes 1-1 \otimes B\right) \\
& =\sum_{\lambda \in Z(g)} \sum_{q}(-1)^{q} \operatorname{Ind}\left(H_{q}(A)(\lambda) \otimes 1-1 \otimes B\right) \\
& =\sum_{\lambda \in Z(g)} \sum_{q}(-1)^{q} \operatorname{Dim}_{\mathbb{C}}\left(H_{q}(g(A), \mathscr{H})(\lambda)\right) \cdot \operatorname{Ind}(\lambda-B) \\
& =-\sum_{\lambda \in Z(g)} \operatorname{Ind}_{\lambda}(g(A)) \cdot \operatorname{Ind}(\lambda-B)
\end{aligned}
$$

where $H_{q}(A) \otimes 1-1 \otimes B$ acts on the Hilbert space $H_{q}(g(A), \mathscr{H}) \otimes \mathscr{G}$. But this is equivalent to the identity in (7.3) and the proposition is proved.

We are now in position to prove the reciprocity formula for local indices.

Theorem 7.6. Let $g: \operatorname{Sp}(A) \cup \mathrm{Sp}(B) \rightarrow \mathbb{C}^{m}$ be holomorphic. Suppose that $g(A)$ and $g(B)$ are Fredholm and that the sets $Z(g) \cap \mathrm{Sp}(A) \cap \mathrm{Sp}_{\mathrm{ess}}(B)$ and $Z(g) \cap$ $\mathrm{Sp}(B) \cap \mathrm{Sp}_{\mathrm{ess}}(A)$ are empty. We then have the identity

$$
\sum_{\mu \in Z(g) \cap \mathrm{Sp}(B)} \operatorname{Ind}(\mu-A) \cdot \operatorname{Ind}_{\mu}(g(B))=\sum_{\lambda \in Z(g) \cap \operatorname{Sp}(A)} \operatorname{Ind}_{\lambda}(g(A)) \cdot \operatorname{Ind}(\lambda-B) .
$$

Proof. By an application of Proposition 7.5 we get the identities

$$
\begin{aligned}
& \operatorname{Ind}((A \otimes 1-1 \otimes B) \oplus(g(A) \otimes 1))=-\sum_{\lambda \in Z(g) \cap \operatorname{Sp}(A)} \operatorname{Ind}_{\lambda}(g(A)) \cdot \operatorname{Ind}(\lambda-B) \text { and } \\
& \operatorname{Ind}((A \otimes 1-1 \otimes B) \oplus(1 \otimes g(B)))=-\sum_{\mu \in Z(g) \cap \operatorname{Sp}(B)} \operatorname{Ind}(\mu-A) \cdot \operatorname{Ind}_{\mu}(g(B))
\end{aligned}
$$

But this entails the result of the theorem since

$$
\operatorname{Ind}((A \otimes 1-1 \otimes B) \oplus(g(A) \otimes 1))=\operatorname{Ind}((A \otimes 1-1 \otimes B) \oplus(1 \otimes g(B)))
$$

by an application of Lemma 7.2.

\section{The local index theorem}

Throughout this section the following conditions will be in effect:

Let $m \geq n$ and let $g: \operatorname{Sp}(A) \rightarrow \mathbb{C}^{m}$ be a holomorphic map on the Taylor spectrum of the commuting tuple $A=\left(A_{1}, \ldots, A_{n}\right)$ of bounded operators such that $g(A)$ is Fredholm. 
It is the aim of this section to compute the local index at a common zero $\lambda \in Z(g)$ in terms of the local degree $\operatorname{deg}_{\lambda}(g)$ and the index $\operatorname{Ind}(A-\lambda)$. It should be emphasized that the techniques applied to prove the global index theorem in Section 6 do not carry over to a proof of the local index theorem. The main problem is that the local index $\operatorname{Ind}_{\lambda}(g(A))$ is not homotopy invariant (there exists a neighborhood $U$ of zero such that $\operatorname{Ind}_{\lambda}(g(A)-z)=0$ for all $\left.z \in U \backslash\{0\}\right)$. Our solution to this problem is to find a Hilbert space equipped with a commuting tuple $D$ (which is tightly related to $g(A)$ ) such that $\operatorname{Ind}(D)=\operatorname{Ind}_{\lambda}(g(A)$ ). To obtain this we will apply what we call "analytic localization". This basically amounts to taking tensor products with the following Hilbert spaces and "restricting to the diagonal" by taking the union with the commuting tuple $A \otimes 1-1 \otimes B$ when computing Koszul homology groups. We will thus rely heavily on the machinery invented in Section 7.

For each $\varepsilon>0$ and each $\lambda \in \mathbb{C}^{n}$, let $\mathscr{G}_{\varepsilon}(\lambda)$ be a Hilbert space and let $B=\left(B_{1}, \ldots, B_{n}\right)$ be a commuting tuple of bounded operators on $\mathscr{G}_{\varepsilon}(\lambda)$ such that the following conditions are satisfied:

(1) The Taylor spectrum of $B$ is included in the closed ball $\mathrm{cl}\left(\mathbb{B}_{\varepsilon}(\lambda)\right)$ in $\mathbb{C}^{n}$ with radius $\varepsilon>0$ and center $\lambda \in \mathbb{C}^{n}$.

(2) The commuting tuple $B-\lambda$ is Fredholm with $\operatorname{Ind}(B-\lambda)=-1$.

An example of a pair $\left(\mathscr{G}_{\varepsilon}(\lambda), B\right)$ with the above properties consists of the Bergman space $H^{2}\left(\mathbb{B}_{\varepsilon}(\mu)\right)$ and the commuting tuple $T_{z}=\left(T_{z_{1}}, \ldots, T_{z_{n}}\right)$ of Toeplitz operators associated with the coordinate functions. See Theorem 9.1.

Lemma 8.1. Let $\lambda \in \mathbb{C}^{n}$ and let $\varepsilon>0$. Suppose that $\operatorname{cl}\left(\mathbb{B}_{\varepsilon}(\lambda)\right) \cap Z(g) \subseteq\{\lambda\}$. Then the commuting tuple $((A \otimes 1-1 \otimes B) \oplus(g(A) \otimes 1))$ is Fredholm on $\mathscr{H} \widehat{\otimes} \mathscr{G}_{\varepsilon}(\lambda)$ with index

$$
\operatorname{Ind}((A \otimes 1-1 \otimes B) \oplus(g(A) \otimes 1))=\operatorname{Ind}_{\lambda}(g(A)) .
$$

Note that $\operatorname{Ind}_{\lambda}(g(A))=0$ whenever $\lambda \notin Z(g)$.

Proof. It follows from Proposition 7.5 and the conditions on $\left(\mathscr{G}_{\varepsilon}(\lambda), B\right)$ that $(A \otimes 1-1 \otimes B) \oplus(g(A) \otimes 1)$ is Fredholm on $\mathscr{H} \widehat{\otimes} \mathscr{G}_{\varepsilon}(\lambda)$ with index

$$
\operatorname{Ind}((A \otimes 1-1 \otimes B) \oplus(g(A) \otimes 1))=-\sum_{\mu \in Z(g)} \operatorname{Ind}_{\mu}(g(A)) \cdot \operatorname{Ind}(\mu-B) .
$$

Since $\operatorname{Ind}(\mu-B)=0$ for all $\mu \notin \operatorname{cl}\left(\mathbb{B}_{\varepsilon}(\lambda)\right)$ and $\operatorname{Ind}(\lambda-B)=-1$ it follows that

$$
-\sum_{\mu \in Z(g)} \operatorname{Ind}_{\mu}(g(A)) \cdot \operatorname{Ind}(\mu-B)=\left\{\begin{array}{ccc}
\operatorname{Ind}_{\lambda}(g(A)) & \text { for } & \lambda \in Z(g) \\
0 & \text { for } & \lambda \notin Z(g) .
\end{array}\right.
$$

This proves the lemma. 
Notation 8.2. Let $h: \operatorname{Sp}(A) \times \mathbb{C}^{n} \rightarrow \mathbb{C}^{n+m}$ denote the holomorphic map defined by $h(z, w)=(z-w, g(z))$.

Remark 8.3. Let $B=\left(B_{1}, \ldots, B_{n}\right)$ be an arbitrary commuting tuple on a Hilbert space $\mathscr{G}$. It follows from Lemma 7.1 that $h((A \otimes 1) \oplus(1 \otimes B))=(A \otimes 1-1 \otimes B)$ $\oplus(g(A) \otimes 1)$.

Theorem 8.4. Suppose that $m>n$. Then $\operatorname{Ind}_{\lambda}(g(A))=0$ for all $\lambda \in \mathbb{C}^{n}$.

Proof. Since $g(A)$ is Fredholm, the set $Z(g)$ is finite. Choose an $\varepsilon>0$ such that $\operatorname{cl}\left(\mathbb{B}_{\varepsilon}(\lambda)\right) \cap Z(g) \subseteq\{\lambda\}$. The preceding remark and Lemma 8.1 imply that $h((A \otimes 1) \oplus(1 \otimes B))$ is Fredholm with

$$
\operatorname{Ind}(h((A \otimes 1) \oplus(1 \otimes B)))=\operatorname{Ind}_{\lambda}(g(A)) .
$$

But Theorem 6.2 implies that

$$
\operatorname{Ind}(h((A \otimes 1) \oplus(1 \otimes B)))=0
$$

since $m+n>2 n$. This proves the theorem.

Recall that $\Omega:=\operatorname{Int}(\operatorname{Sp}(A))$ denotes the interior of the Taylor spectrum of $A$.

Lemma 8.5. Suppose that $\lambda \in \Omega \cap Z(g)$ and that $n=m$. Then $\operatorname{deg}_{\lambda}(g)=$ $\operatorname{deg}_{(\lambda, \lambda)}(h)$.

Note that $h(z, w)=0 \Leftrightarrow(z=w$ and $g(z)=0)$, thus $Z(h)=\{(\mu, \mu) \mid \mu \in$ $Z(g)\}$ is a finite set. It follows in particular that $\operatorname{deg}_{(\lambda, \lambda)}(h)$ is well-defined.

Proof. Recall from Lemma 6.5 that $\operatorname{deg}_{(\lambda, \lambda)}(h)=\mathcal{O}_{(\lambda, \lambda)} / h \mathcal{O}_{(\lambda, \lambda)}$ where $h \mathcal{O}_{(\lambda, \lambda)}$ denotes the ideal generated by the coordinates of $h$.

The map $\alpha: \mathcal{O}_{(\lambda, \lambda)} /(z-w) \mathcal{O}_{(\lambda, \lambda)} \rightarrow \mathcal{O}_{\lambda}$ given by $f \mapsto f \circ \Delta$, where $\Delta: \mathbb{C}^{n} \rightarrow \mathbb{C}^{2 n}$ is the diagonal map $\Delta(z):=(z, z)$, is an isomorphism of unital algebras over $\mathbb{C}$. The inverse is given by $f \mapsto f \circ p_{n}$, where $p_{n}: \mathbb{C}^{2 n} \rightarrow \mathbb{C}^{n}$ is the projection onto the first $n$ factors.

It follows in particular that $\alpha$ induces an isomorphism between the unital $\mathbb{C}$ algebras

$$
\mathcal{O}_{(\lambda, \lambda)} / h \mathcal{O}_{(\lambda, \lambda)}=\mathcal{O}_{(\lambda, \lambda)} /\left((z-w) \mathcal{O}_{(\lambda, \lambda)}+\left(g \circ p_{n}\right) \mathcal{O}_{(\lambda, \lambda)}\right) \text { and } \mathcal{O}_{\lambda} / g \mathcal{O}_{\lambda} .
$$

This proves the lemma.

The main result of this paper can now be stated and proved. It expresses the local index at a point in terms of the local degree of the symbol $g$ and the locally constant index function of the variables $A$.

Theorem 8.6. Suppose that $g(A)$ is Fredholm, that $n=m$, and that $\lambda \in Z(g)$. The local index at $\lambda$ is then given by

$$
\operatorname{Ind}_{\lambda}(g(A))=\operatorname{deg}_{\lambda}(g) \cdot \operatorname{Ind}(\lambda-A)
$$


Remark that $\operatorname{deg}_{\lambda}(g) \cdot \operatorname{Ind}(\lambda-A)$ is understood to be zero when $\lambda \in \partial \operatorname{Sp}(A) \cap$ $Z(g)$ even though the local degree $\operatorname{deg}_{\lambda}(g)$ need not be well-defined in this case. We do however have that $\operatorname{Ind}(\lambda-A)=0$.

Proof. Choose an $\varepsilon>0$ such that $\operatorname{cl}\left(\mathbb{B}_{\varepsilon}(\lambda)\right) \cap Z(g)=\{\lambda\}$. It follows by Lemma 8.1 and Remark 8.3 that $h((A \otimes 1) \oplus(1 \otimes B))$ is Fredholm on $\mathscr{H} \widehat{\otimes} \mathscr{G}_{\varepsilon}(\lambda)$ with

$$
\operatorname{Ind}(h((A \otimes 1) \oplus(1 \otimes B)))=\operatorname{Ind}_{\lambda}(g(A)) .
$$

Let $C:=(A \otimes 1) \oplus(1 \otimes B)$ and let $W:=\operatorname{Int}(\operatorname{Sp}(C))$. The global index theorem 6.6 implies that

$$
\operatorname{Ind}(h(C))=\sum_{\mu \in W \cap Z(h)} \operatorname{deg}_{\mu}(h) \cdot \operatorname{Ind}(\mu-C) .
$$

By Lemma 7.3 we have that $(\lambda, \lambda)-C$ is Fredholm with

$$
\operatorname{Ind}((\lambda, \lambda)-C)=-\operatorname{Ind}(\lambda-A) \cdot \operatorname{Ind}(\lambda-B)=\operatorname{Ind}(\lambda-A) .
$$

By Lemma 7.1 the open set $W$ is included in $\Omega \times \mathbb{B}_{\varepsilon}(\lambda)$. Furthermore, the set of zeroes of $h: \operatorname{Sp}(C) \rightarrow \mathbb{C}^{2 n}$ is contained in the diagonal $\Delta(Z(g))=\{(v, v) \mid v \in$ $Z(g)\}$. It follows that

$$
W \cap Z(h) \subseteq\left(\Omega \times \mathbb{B}_{\varepsilon}(\lambda)\right) \cap \Delta(Z(g)) \subseteq\{(\lambda, \lambda)\} .
$$

We now have two cases: Either $(\lambda, \lambda) \notin W$ or $(\lambda, \lambda) \in W$.

Suppose first that $(\lambda, \lambda) \notin W=\operatorname{Int}(\operatorname{Sp}(C))$. Then $0=\operatorname{Ind}((\lambda, \lambda)-C)=$ $\operatorname{Ind}(\lambda-A)$. But this implies that both sides of the desired identity $\operatorname{Ind}_{\lambda}(g(A))=$ $\operatorname{deg}_{\lambda}(g) \cdot \operatorname{Ind}(\lambda-A)$ is zero.

Suppose therefore that $(\lambda, \lambda) \in W$. Notice that $h(\lambda, \lambda)=0$ since $\lambda \in Z(g)$ by assumption. Lemma 8.5 entails that $\operatorname{deg}_{(\lambda, \lambda)}(h)=\operatorname{deg}_{\lambda}(g)$. It can thus be concluded that

$\operatorname{Ind}_{\lambda}(g(A))=\operatorname{Ind}(h(C))=\operatorname{deg}_{(\lambda, \lambda)}(h) \cdot \operatorname{Ind}((\lambda, \lambda)-C)=\operatorname{deg}_{\lambda}(g) \cdot \operatorname{Ind}(\lambda-A)$.

This proves the theorem.

\section{Examples}

9.1. Bergman space. Let $\Omega \subseteq \mathbb{C}^{n}$ be a bounded open set. We let $L^{2}(\Omega)$ denote the Hilbert space of square integrable functions on $\Omega$ (w.r.t. Lebesgue measure). The closed subspace of holomorphic square integrable functions will be denoted by $H^{2}(\Omega)$. This is the Bergman-space associated with $\Omega$. 
For each $i \in\{1, \ldots, n\}$ we have the Toeplitz operator $T_{z_{i}} \in \mathscr{L}\left(H^{2}(\Omega)\right)$ given by multiplication with the $i^{\text {th }}$ coordinate function $z_{i}: \Omega \rightarrow \mathbb{C}$. We will then focus on the commuting tuple of Toeplitz operator $T_{z}:=\left(T_{z_{1}}, \ldots, T_{z_{n}}\right)$. The next result can be found as [18, Theorem 1.3].

Theorem 9.1. Suppose that $\Omega$ is pseudoconvex and that $\partial \Omega=\partial(\operatorname{cl}(\Omega))$. Then the following assertions are valid:

(1) The spectrum for $T_{z}$ is the closure of $\Omega, \operatorname{Sp}\left(T_{z}\right)=\operatorname{cl}(\Omega)$.

(2) The essential spectrum for $T_{z}$ is the boundary of $\Omega, \operatorname{Sp}_{\mathrm{ess}}\left(T_{z}\right)=\partial \Omega$.

(3) For each $\lambda \in \Omega$ the Koszul homology of $T_{z}-\lambda$ is concentrated in degree 0 and $\operatorname{Ind}\left(T_{z}-\lambda\right)=-1$.

Let $g: \operatorname{Sp}\left(T_{z}\right)=\operatorname{cl}(\Omega) \rightarrow \mathbb{C}^{n}$ be a holomorphic function. We then have the commuting tuple of Toeplitz operators $T_{g}=\left(T_{g_{1}}, \ldots, T_{g_{n}}\right)$ where each $T_{g_{i}} \in$ $\mathscr{L}\left(H^{2}(\Omega)\right)$ acts by multiplication with $g_{i}$.

As an application of our local index theorem we can now obtain the following.

Corollary 9.2. Suppose that $\Omega$ is pseudoconvex and that $\partial \Omega=\partial(\operatorname{cl}(\Omega))$. Suppose furthermore that $0 \notin g(\partial \Omega)$ and that $\lambda \in Z(g)$. Then the commuting tuple $T_{g}$ is Fredholm and the local index at $\lambda$ is given by

$$
\operatorname{Ind}_{\lambda}\left(T_{g}\right)=-\operatorname{deg}_{\lambda}(g) .
$$

In particular we have that $\operatorname{Ind}\left(T_{g}\right)=-\operatorname{deg}(0, g, \Omega)$.

Proof. By Theorem 8.6 and Theorem 9.1 we only need to prove that $T_{f}=f\left(T_{z}\right)$ for each holomorphic function $f: \operatorname{cl}(\Omega) \rightarrow \mathbb{C}$. Let $\mu \in \Omega$ and let $\varepsilon_{\mu}: H^{2}(\Omega) \rightarrow \mathbb{C}$ denote the functional given by evaluation at $\mu$. Let $\xi \in H^{2}(\Omega)$. It is then sufficient to show that $\varepsilon_{\mu}\left(f\left(T_{z}\right)(\xi)\right)=f(\mu) \cdot \varepsilon_{\mu}(\xi)$. But this follows immediately from Proposition 2.12 and [19, Theorem 3.16].

9.2. Hardy space. In this section we will show that our local index theorem also applies to the Hardy space over the polydisc. Let us briefly recall some definitions. For general information we refer to [14].

We let $\mathbb{C}\left[z_{1}, \ldots, z_{n}\right]$ denote the $\mathbb{C}$-algebra of polynomials in the coordinate functions $z_{1}, \ldots, z_{n}: \mathbb{T}^{n} \rightarrow \mathbb{C}$ on the $n$-torus. The Hardy space over the polydisc is then defined as the closure of $\mathbb{C}\left[z_{1}, \ldots, z_{n}\right]$ inside the Hilbert space $L^{2}\left(\mathbb{T}^{n}\right)$ of square integrable functions on the $n$-torus. We will denote this Hardy space by $H^{2}\left(\mathbb{T}^{n}\right)$.

The coordinate functions $z_{1}, \ldots, z_{n}$ act by multiplication on $H^{2}\left(\mathbb{T}^{n}\right)$ giving rise to a commuting tuple $T_{z}:=\left(T_{z_{1}}, \ldots, T_{z_{n}}\right)$ of Toeplitz operators. The next theorem can easily be deduced from [2, Theorem 5]:

Theorem 9.3. The spectrum of $T_{z}$ is the closed polydisc, $\operatorname{Sp}\left(T_{z}\right)=\mathbb{D}^{n}$ whereas the essential spectrum of $T_{z}$ is the boundary, $\operatorname{Sp}_{\mathrm{ess}}\left(T_{z}\right)=\partial \mathbb{D}^{n}$. For each point $\lambda \in U^{n}$ the Koszul homology of $T_{z}-\lambda$ is concentrated in degree 0 and $\operatorname{Ind}\left(T_{z}-\lambda\right)=-1$. 
Let $g: \operatorname{Sp}\left(T_{z}\right)=\mathbb{D}^{n} \rightarrow \mathbb{C}^{n}$ be a holomorphic function. We then have the commuting tuple of Toeplitz operators $T_{g}=\left(T_{g_{1}}, \ldots, T_{g_{n}}\right)$ where $T_{g_{i}}$ acts by multiplication with $g_{i}$ on $H^{2}\left(\mathbb{T}^{n}\right)$. As in the case of the Bergman spaces it is not hard to see that $T_{g}=g\left(T_{z}\right)$ where $g\left(T_{z}\right)$ is constructed using the analytic functional calculus. An application of our local index theorem now yields the following.

Corollary 9.4. Suppose that $0 \notin g\left(\partial \mathbb{D}^{n}\right)$ and that $\lambda \in Z(g)$. Then the commuting tuple $T_{g}$ is Fredholm and the local index at $\lambda$ is given by

$$
\operatorname{Ind}_{\lambda}\left(T_{g}\right)=-\operatorname{deg}_{\lambda}(g) .
$$

In particular we have that $\operatorname{Ind}\left(T_{g}\right)=-\operatorname{deg}\left(0, g, U^{n}\right)$ where $U:=\mathbb{D}^{\circ}$ denotes the open disc.

\section{References}

[1] R. Carey and J. Pincus, Joint torsion of Toeplitz operators with $H^{\infty}$ symbols, Integral Equations Operator Theory 33 (1999), no. 3, 273-304. Zbl 0951.47030 MR 1671481

[2] R. E. Curto, Fredholm and invertible $n$-tuples of operators. The deformation problem, Trans. Amer. Math. Soc. 266 (1981), no. 1, 129-159. Zbl 0457.47017 MR 613789

[3] D. Eisenbud and H. I. Levine, An algebraic formula for the degree of a $C^{\infty}$ map germ, Ann. of Math. (2) 106 (1977), no. 1, 19-44, With an appendix by Bernard Teissier, "Sur une inégalité à la Minkowski pour les multiplicités”. Zbl 0398.57020 MR 467800

[4] J. Eschmeier and M. Putinar, Spectral decompositions and analytic sheaves, London Mathematical Society Monographs. New Series, vol. 10, The Clarendon Press Oxford University Press, New York, 1996, Oxford Science Publications. Zbl 0855.47013 MR 1420618

[5] A. S. Faŭnšteĭn, The joint essential spectrum of a family of linear operators, Funktsional. Anal. i Prilozhen. 14 (1980), no. 2, 83-84. MR 575225

[6] X. Fang, The Fredholm index of a pair of commuting operators, Geom. Funct. Anal. 16 (2006), no. 2, 367-402. Zbl 1105.47008 MR 2231467

[7] H. Grauert and R. Remmert, Coherent analytic sheaves, Grundlehren der Mathematischen Wissenschaften [Fundamental Principles of Mathematical Sciences], vol. 265, Springer-Verlag, Berlin, 1984. Zbl 0537.32001 MR 755331

[8] J. Kaad, Joint torsion of several commuting operators, Adv. Math. 229 (2012), no. 1, 442-486. Zbl 1248.47006 MR 2854180

[9] K. Kodaira, Complex manifolds and deformation of complex structures, Grundlehren der Mathematischen Wissenschaften [Fundamental Principles of Mathematical Sciences], vol. 283, Springer-Verlag, New York, 1986, Translated from the Japanese by Kazuo Akao, With an appendix by Daisuke Fujiwara. Zbl 0581.32012 MR 815922

[10] R. N. Levy, Notes on the taylor joint spectrum of commuting operators, Banach Center Publ. VIII; Operator Theory (1981), 321-332. Zbl 0496.47017 MR 738292 
[11] R. N. Levy, Algebraic and topological $K$-functors of commuting $n$-tuple of operators, J. Operator Theory 21 (1989), no. 2, 219-253. Zbl 0704.46047 MR 1023314

[12] M. Putinar, Algebraic properties of Fredholm systems, Proceedings of the Fourth Conference on Operator Theory (Timişoara/Herculane, 1979) (Timişoara), Univ. Timişoara, 1980, pp. 187-198. Zbl 0567.47013 MR 657802

[13] M. Putinar, Base change and the Fredholm index, Integral Equations Operator Theory 8 (1985), no. 5, 674-692. Zbl 0582.47017 MR 813356

[14] W. Rudin, Function theory in polydiscs, W. A. Benjamin, Inc., New York-Amsterdam, 1969. Zbl 0177.34101 MR 255841

[15] J. T. Schwartz, Nonlinear functional analysis, Gordon and Breach Science Publishers, New York, 1969, Notes by H. Fattorini, R. Nirenberg and H. Porta, with an additional chapter by Hermann Karcher, Notes on Mathematics and its Applications. Zbl 0203.14501 MR 433481

[16] J.-P. Serre, Local algebra, Springer Monographs in Mathematics, Springer-Verlag, Berlin, 2000, Translated from the French by CheeWhye Chin and revised by the author. Zbl 0959.13010 MR 1771925

[17] J.-P. Serre, Lie algebras and Lie groups, Lecture Notes in Mathematics, vol. 1500, Springer-Verlag, Berlin, 2006, 1964 lectures given at Harvard University, Corrected fifth printing of the second (1992) edition. Zbl 0742.17008 MR 2179691

[18] N. Salinas, A. Sheu, and H. Upmeier, Toeplitz operators on pseudoconvex domains and foliation $C^{*}$-algebras, Ann. of Math. (2) 130 (1989), no. 3, 531-565. Zbl 0708.47021 MR 1025166

[19] J. L. Taylor, The analytic-functional calculus for several commuting operators, Acta Math. 125 (1970), 1-38. Zbl 0233.47025 MR 271741

[20] J. L. Taylor, A joint spectrum for several commuting operators, J. Functional Analysis 6 (1970), 172-191. Zbl 0233.47024 MR 268706

[21] C. A. Weibel, An introduction to homological algebra, Cambridge Studies in Advanced Mathematics, vol. 38, Cambridge University Press, Cambridge, 1994. Zbl 0797.18001 MR 1269324

Received 24 October, 2012; revised 31 July, 2013

J. Kaad, Institut de Mathématiques de Jussieu, Université de Paris VII, 175 rue du Chevaleret, 75013 Paris, France

E-mail: jenskaad@hotmail.com

R. Nest, Department of Mathematical Sciences, Universitetsparken 5, DK-2100 Copenhagen $\varnothing$, Denmark

E-mail: rnest@math.ku.dk 